Y. Kato

Nagoya Math. J.

Vol. 129 (1993), 53-95

\title{
VARIATIONAL INEQUALITIES OF BINGHAM TYPE IN THREE DIMENSIONS
}

\author{
YOSHIO KATO
}

\section{Introduction}

The flow of Bingham type through a domain $\Omega$ in the $d$-th dimensional space $\mathbf{R}^{d}(d \geq 2)$ during the time $(0, T)$ is a flow of an incompressible visco-plastic fluid governed by the equations for a velocity vector $u=\left(u^{1}, \ldots, u^{d}\right)$ and a stress tensor $\sigma=\left(\sigma_{i j}\right)_{i, j=1}^{d}$ :

$$
\begin{array}{ll}
\frac{\partial u}{\partial t}+u \cdot \nabla u=f+\nabla \sigma & \\
\nabla \cdot u=0 & \text { in } \Omega \times(0, T)
\end{array}
$$

and by the constituent law:

$$
\begin{array}{ll}
\sigma^{D}=\left\{\eta(|D|)+\frac{g}{|D|}\right\} D & \text { when } D \neq 0 \\
\left|\sigma^{D}\right| \leq g & \text { when } D=0
\end{array}
$$

which is equivalent to

$$
\eta(|D|) D= \begin{cases}\left(1-g /\left|\sigma^{D}\right|\right) \sigma^{D} & \text { when }\left|\sigma^{D}\right|>g \\ 0 & \text { when }\left|\sigma^{D}\right| \leq g\end{cases}
$$

where $\sigma^{D}=\sigma+\pi I_{d}$ is the deviation of $\sigma$ (i.e., $\pi=-\operatorname{tr}(\sigma) / d$ is the pressure), $g$ the yield limit, $D=D(u)$ a tensor of strain velocity with components:

$$
D_{i j}(u)=\frac{1}{2}\left(\nabla_{i} u^{j}+\nabla_{j} u^{i}\right) \text { with } \nabla_{i}=\partial / \partial x_{i},
$$

$|\sigma|$ the length defined by

$$
|\sigma|=(\sigma \cdot \sigma)^{1 / 2}, \quad \sigma \cdot \tau=\sigma_{i j} \tau_{i j},
$$

$u \cdot \nabla=u^{i} \nabla_{i},(\nabla \cdot \sigma)_{i}=\nabla_{j} \sigma_{i j}$ and $\nabla \cdot u=\nabla_{i} u^{i}=\operatorname{div} u$, the summation convention concerning repeated indices being used.

Recieved July 22, 1991. 
In the present paper we consider a fluid with viscosity $\eta(|D|)$ such that $\lambda \eta(\lambda)$ is a nondecreasing function in $\lambda \geq 0$ satisfying

$$
c_{1} \lambda^{p-1} \leq \lambda \eta(\lambda) \leq c_{2} \lambda^{p-1}, \quad \lambda \geq 0
$$

for some positive constants $c_{1}, c_{2}$ and $p>1$. The various interesting examples of $\eta(\lambda)$ may be found in Astarita-Marrucci [1]. Introducing a convex functional of $u$ :

$$
\varphi(u)=\int_{\Omega} d x \int_{0}^{|D(u)|}(\lambda \eta(\lambda)+g) d \lambda,
$$

we can deduce after Duvaut-Lions [5] the equations (0.1)-(0.2) subject to the boundary condition $u=0$ to the evolution inequality

$$
\begin{gathered}
\int_{\Omega}\left(u^{\prime}(t)+B(u(t)) \cdot(v-u(t)) d x+\varphi(v)-\varphi(u(t))\right. \\
\geq \int_{\Omega} f(t) \cdot(v-u(t)) d x
\end{gathered}
$$

for all $t \in(0, T)$ and all $v$ such that $\nabla \cdot v=0$ in $\Omega$ and $v=0$ on the boundary $\partial \Omega$ of $\Omega$, where $u^{\prime}=d u / d t$ and $B(u)=u \cdot \nabla u$. The inequality (0.4) is called to be of Bingham type if $g>0$.

The problem we consider here is to find a solution $u(t)=u(x, t)$ of inequality (0.4) of Bingham type satisfying the boundary condition

$$
u(x, t)=0 \text { on } \partial \Omega \times(0, T)
$$

and the initial condition

$$
u(x, 0)=u_{0}(x) \text { in } \Omega .
$$

The fluid which is obeyed by $(0.2)$ with constant viscosity $\eta$ is called a Bingham fluid, whose flow was first studied by Duvaut-Lions $[5,6]$ introducing a variational inequality such as (0.4). They obtained, among other things, a weak solution (for the definition see Theorem 1). In Naumann-Wulst [13,14] strong solutions (for the definition see Corollary 1 ) were looked for in the case $\eta(\lambda)=$ $\lambda^{p-2}$, $(\sqrt{97}-1) / 4 \leq p<3$, under the condition that $\Omega$ is a smooth and bounded domain in $\mathbf{R}^{3}$. The existence of a strong solution for a Bingham fluid was investigated by Kim $[7,8]$ in the plane as well as in the third dimensional bounded domain.

The main result of this paper consists of three theorems. Theorem 1 is concerned with the existence of weak solutions to the initial-boundary value problem $(0.4) \sim(0.6)$ with $p>6 / 5$ where $\varphi$ is allowed to depend explicitly on $t$. As a 
corollary we obtain strong solutions for $p \geq 11 / 5$ (see Corollary 1). This result is a slight improvement of a result of Naumann-Wulst [14, Theorem 1.1 (i)]. In Theorem 2 we derive the energy inequality of strong form, provided that $\Omega$ is an exterior domain and $\eta(\lambda)=\mu \lambda^{p-2}$ with positive constant $\mu$ and $p \geq 9 / 5$. The regularity of velocity field $u$ of Bingham fluid with variable viscosity and yield limit will be investigated in Theorem 3. This is nothing but a simple extension of the result of Kim [8].

The distinctive feature of the present paper is to construct Yosida's approximation $\mathscr{L}_{n}=n\left\{1-\left(1+\frac{1}{n} L_{n}\right)^{-1}\right\}$ of a multivalued operator $L_{n}(v)$ $=e_{n}(v)+B(v)+\partial \varphi(v)$ which is regularized by adding the term $e_{n}(v)=$ $-\xi_{n} \exp \left(\lambda_{n}\|\nabla v\|^{c}\right) \Delta v$ where $c>4$ and $\xi_{n}, \lambda_{n} \rightarrow 0$ as $n \rightarrow \infty$. In fact, it is proved in Section 3 that the inverse of an operator $\left(1+\frac{1}{n} L_{n}\right)$ exists. The evolution equation $u_{n}^{\prime}(t)+\mathscr{L}_{n}\left(t, u_{n}(t)\right)=f_{n}(t)$ which approximates $(0.4)$ will be solved by the method of successive approximation. A weak solution which is seeked for in Theorem 1 will be found in Section 4 as a limit of a subsequence of $\left\{u_{n}\right\}$.

The proof of Theorem 2 is achieved in Section 5 by taking a test function of the form $\operatorname{rot}\left\{\zeta_{\lambda}\left(F_{\lambda} *\left(\zeta_{\lambda} \operatorname{rot} u_{n}\right)\right)\right\}(\lambda \rightarrow 0)$ where $F_{\lambda}$ denotes a fundamental solution of operator $\lambda-\Delta$ and $\zeta_{\lambda}$ a cut-off function such that $\zeta_{\lambda}(x)=1$ for $|x|>2 / \lambda$ and $=0$ for $|x|>1 / \lambda$. This device for the proof comes into action thanks to the plastic term $g|D(u)|$. For the Navier-Stokes equation where $p=2$ and $g=0$ we refer to Miyakawa-Sohr [11].

Theorem 3 is able to be applied to problems of heat transfer in a Bingham fluid with viscosity and yield limit depending on the temperature, which will be investigated elsewhere.

We devote Section 1 to preparations for the present study. Theorems $1 \sim 3$ are stated in Section 2, along with three corollaries and four remarks where Theorems $1 \sim 3$ are examined in the case that $d=2$. Sections $4 \sim 6$ are devoted to the proof of Theorems $1-3$, respectively.

\section{§1. Preliminaries}

By $\mathscr{V}$ we denote the set of $v=\left(v^{1}, \ldots, v^{d}\right) \in C_{0}^{\infty}\left(\mathbf{R}^{d}\right)^{d}$ such that $\nabla \cdot v=0$ everywhere and by $L^{p}(1 \leq p \leq \infty)$ the set of all $L^{p}$-function from $\mathbf{R}^{d}$ $(d \geq 2)$ into $\mathbf{R}$ equipped with the usual $L^{p}$-norm $\|\cdot\|_{p}$. Especially, we simply write $\|\cdot\|_{2}=\|\cdot\|$. Further, the following abbreviations are used: $\|v\|_{p}=\||v|\|_{p}$, 
$\|\nabla v\|_{p}=\||\nabla v|\|_{p}$ and $\|D(v)\|_{p}=\||D(v)|\|_{p}$ for vector field $v$, where $\nabla v$ and $D(v)$ denote tensors with components $\nabla_{i} v^{j}$ and $D_{i j}(v)=\nabla_{i} v^{j}+\nabla_{j} v^{i}$, and $|\cdot|$ respective length with respect to the euclidian metric.

We start with stating the two fundamental inequalities.

Korn's inequality. For any $p \in(1 . \infty)$ there exists a positive constant $K_{p}$ such that

$$
\|\nabla v\|_{p} \leq K_{p}\|D(v)\|_{p}, \quad v \in C_{0}^{\infty}\left(\mathbf{R}^{d}\right)^{d} .
$$

Sobolev's inequality: For any $p \in[1, d)$ there exists a positive constant $S_{p}$ such that

$$
\|v\|_{p^{*}} \leq S_{p}\|D(v)\|_{p}, \quad v \in C_{0}^{\infty}\left(\mathbf{R}^{d}\right)^{d},
$$

where $p^{*}=d p /(d-p)$

For the proof of (1.1) we refer to Mosolov-Mjasnikov [12] and its bibliography. Combining (1.1) and the usual Sobolev inequality (see Berger [2]), we immediately obtain (1.2) for $p, 1<p<d$. The inequality (1.2) with $p=1$ has been proved by Strauss [16].

The following proposition is nothing but a straightforward extension of the result of Renardy [15].

Proposition 1.1. There exists a sequence of operators $T_{\varepsilon, \lambda, \mu}(\varepsilon, \lambda, \mu>0)$; $u \rightarrow u_{\varepsilon, \lambda, \mu}=T_{\varepsilon, \lambda, \mu} u$ of $L_{\sigma}^{q}(1 \leq q<\infty)$ into $\mathscr{V}$ such that

(i ) $u_{\varepsilon, \lambda, \mu} \rightarrow u$ in $L^{q}$,

(ii) $\nabla u_{\varepsilon, \lambda, \mu} \rightarrow \nabla u \quad$ in $L^{p}$, if $\nabla_{i} u^{j} \in L^{\mathrm{p}}(1 \leq i, j \leq d)$ and $p>1$,

and

(iii) $D\left(u_{\varepsilon, \lambda, \mu}\right) \rightarrow D(u)$ in $L^{r}$, if $D_{i j}(u) \in L^{r}(1 \leq i, j \leq d)$ for $r \geq 1$ such that $1 / r-1 / q \leq 2 / d$,

as $\mu \rightarrow 0, \lambda \rightarrow 0$ and $\varepsilon \rightarrow 0$, one after another, where

$$
\begin{aligned}
& L_{\sigma}^{q}=\left\{u \in\left(L^{q}\right)^{d} ; \nabla \cdot u=0\right\} \text { for } q>1, \\
& L_{\sigma}^{1}=\left\{u \in\left(L^{1}\right)^{d} ; \nabla \cdot u=0 \text { and } \int u d x=0\right\} .
\end{aligned}
$$

Proof. For a $C^{\infty}$-function $\xi(t)$ on $[0, \infty)$ such that $\xi(t)=1$ for $t<1,=0$ for $t>2$ and $0 \leq \xi(t) \leq 1$ we introduce two functions on $\mathbf{R}^{d}$ : 


$$
\eta(x)=\xi(|x|) \text { and } \rho(x)=\eta(x) / \int \eta(x) d x,
$$

and a cut-off function:

$$
\phi(x)=1 / \operatorname{vol}\left(B_{1}\right) \text { on } B_{1} \text { and }=0 \text { outside } B_{1},
$$

where and in what follows $B_{R}$ denotes an open ball of radius $R$ with center the origin. For positive numbers $\lambda, \mu$, $\varepsilon$ we set

$$
\eta_{\mu}(x)=\eta(\mu x), \rho_{\varepsilon}(x)=\varepsilon^{-d} \rho(x / \varepsilon) \text { and } \phi_{\lambda}(x)=\lambda^{d} \phi(\lambda x) .
$$

Denoting by $G$ the fundamental solution of the laplacian, we define

$$
G_{\varepsilon, \lambda}=G *\left(\delta-\phi_{\lambda}\right) * \rho_{\varepsilon},
$$

where $f * g$ denotes the convolution of $f$ and $g$, and $\delta$ the Dirac function. The use of Fourier transformation asserts that $G_{\varepsilon, \lambda}$ is rapidly decreasing along with its all derivatives. In the course of the proof we also use the well-known inequality in the literature:

$$
\|f * g\|_{r} \leq\|f\|_{p}\|g\|_{q} \quad(1 \leq p, q, r \leq \infty \text { and } 1 / p+1 / q=1+1 / r)
$$

and the lemma due to Renardy [15]: Suppose that $f \in L^{r}(1 \leq r<\infty)$ and further assume that $\int f(x) d x=0$ in the case $r=1$. Then, we have

$$
\phi_{\lambda} * f \rightarrow 0 \text { in } L^{r} \text { as } \lambda \rightarrow 0 \text {. }
$$

We now define an operator $T_{\varepsilon, \lambda, \mu}$ of $L_{\sigma}^{q}(q \geq 1)$ into $\mathscr{V}$ :

$$
u_{\varepsilon, \lambda, \mu}^{j}=\left(T_{\varepsilon, \lambda, \mu} u\right)^{j}=-\nabla_{k}\left\{\eta_{\mu}\left(G_{\varepsilon, \lambda} * \operatorname{rot}_{k j} u\right)\right\},
$$

where $\operatorname{rot}_{k j} u=\nabla_{k} u^{j}-\nabla_{j} u^{k}$. A simple calculation leads to

$$
u_{\varepsilon, \lambda, \mu}^{j}=\eta_{\mu}\left\{\left(\delta-\phi_{\lambda}\right) * \rho_{\varepsilon} * u^{j}\right\}-\left(\nabla_{k} \eta_{\mu}\right)\left(G_{\varepsilon, \lambda} * \operatorname{rot}_{k j} u\right)
$$

and

$$
\begin{aligned}
\nabla_{i} u_{\varepsilon, \lambda, \mu}^{j} & =\eta_{\mu}\left\{\left(\delta-\phi_{\lambda}\right) * \rho_{\varepsilon} * \nabla_{i} u^{j}\right\} \\
& -\left\{\nabla_{i} \eta_{\mu}\left(\nabla_{k} G_{\varepsilon, \lambda} * \nabla_{k} u^{j}\right)+\nabla_{k} \eta_{\mu}\left(\nabla_{i} G_{\varepsilon, \lambda} * \operatorname{rot}_{k j} u\right)\right\} \\
& -\left(\nabla_{i} \nabla_{k} \eta_{\mu}\right)\left(G_{\varepsilon, \lambda} * \operatorname{rot}_{k j} u\right) \equiv a_{i j}+b_{i j}+c_{i j} .
\end{aligned}
$$

The assertions (i) and (ii) immediately follow from the above two equalities. To prove (iii) we derive from (1.7)

$$
\begin{aligned}
D_{i j}\left(u_{\varepsilon, \lambda, \mu}\right) & =\eta_{\mu}\left\{\left(\delta-\phi_{\lambda}\right) * \rho_{\varepsilon} * D_{i j}(u)\right\}+\left(b_{i j}+b_{j i}\right) / 2+\left(c_{i j}+c_{j i}\right) / 2 \\
& =A_{i j}+B_{i j}+C_{i j} .
\end{aligned}
$$


It is easy to see by (1.5) that $A_{i j} \rightarrow D_{i j}(u)$ in $L^{r}$. The use of (1.4) and the identity

$$
\nabla_{i} \nabla_{j} u^{k}=\nabla_{j} D_{k i}(u)+\nabla_{i} D_{j k}(u)-\nabla_{k} D_{i j}(u)
$$

guarantee us that $B_{i j} \rightarrow 0$ in $L^{r}$ as $\mu \rightarrow 0$.

Our final goal is to show that $C_{i j} \rightarrow 0$ in $L^{r}$ as $\mu \rightarrow 0$. To do so let us first remark that $C_{i j}$ is represented as a linear combination of terms of the form $U=\left(\nabla_{j} \nabla_{k} \eta_{\mu}\right)\left(G_{\varepsilon, \lambda} * \nabla u\right)$. Let us assume $r \geq q$. The inequality (1.4) then leads to

$$
\|U\|_{r} \leq C \mu^{2}\left\|\nabla G_{\varepsilon, \lambda}\right\|_{p}\|u\|_{q}, \quad p \geq 1 .
$$

Thus, $\|U\|_{r} \rightarrow 0$ as $\mu \rightarrow 0$. If $r<q$, we use Hölder's inequality:

$$
\|U\|_{r} \leq C \mu^{2-d / p}\left(\int_{|x| \geq 2 / \mu}\left|\nabla G_{\varepsilon, \lambda} * u\right|^{q} d x\right)^{1 / q},
$$

where $1 / p+1 / q=1 / r$. Application of (1.4) with $p=1$ implies $\nabla G_{\varepsilon, \lambda} * u \in L^{q}$ and our assumption on $q$ and $r$ yields $2-d / p \geq 0$. Consequently, $U \|_{r} \rightarrow 0$ as $\mu \rightarrow 0$.

Q. E. D.

In this section we always assume

$\Omega$ an arbitrary domain in $\mathbf{R}^{d}(d \geq 2)$,

$H$ the closure of $\mathscr{V}(\Omega)=\{v \in \mathscr{V}$; supp $v \subset \Omega\}$ by norm $\|v\|$.

and

$$
Y_{p}\left(\mathbf{R}^{d}\right) \text { the closure of } \mathscr{V} \text { by norm }\|D(v)\|_{p}(p \geq 1) \text {. }
$$

It is easy to see that $Y_{p}\left(\mathbf{R}^{d}\right)$ is imbedded in $L_{\text {loc }}^{p}\left(\mathbf{R}^{d}\right)^{d}$. Therefore, we may introduce the Banach spaces which play important parts in the paper:

$$
V_{p}=Y_{p}\left(\mathbf{R}^{d}\right) \cap H \text { equipped with norm }\|v\|_{V_{p}}=\|D(v)\|_{p}+\|v\|
$$

and, setting $V=V_{2}$,

$$
W_{p}=V_{p} \cap V \text { equipped with norm }\|v\|_{W_{p}}=\|v\|_{V_{p}}+\|v\|_{V} .
$$

It is evident that every function in $V_{p}$ vanishes outside of the closure $\bar{\Omega}$ of $\Omega$. According to Lions $\left[9\right.$, p.6], we can assert that $V_{p}$ is separable for any $p \geq 1$ and further reflexive if $p>1$ and that $V_{p} \subset H \subset V_{p}^{\prime}$, where $H$ is identified with its dual $H^{\prime}$, each space is dense in the following and the injections are one to one and continuous. These assertions hold true for $W_{p}$ as well.

There are given two separable Banach spaces $X$ and $Y$ such that $X \subset Y \subset H$, where each space is dense in the following and the injections are one to one and continuous. Denoting by $\langle,\rangle_{X}$ the duality between $X^{\prime}$ and $X$, it is easily verified 
that $\langle f, u\rangle_{X}=\langle f, u\rangle_{Y}$ for $u \in X$ and $f \in Y^{\prime}$. So it will be allowed to write it as $\langle f, u\rangle$ without any confusion. In particular, $\langle f, u\rangle$ means the inner product in $H$ if $u, f \in H$.

Lemma 1.1. Suppose that $2 \leq d \leq 4$.

(i) For all $r \geq 1$ we have $V_{r}=\left\{u \in H ; D_{i j}(u) \in L^{\mathrm{r}}(1 \leq i, j \leq d)\right\}$.

(ii) For all $q, r \in[1, p]$ such that $q<d$ we have $V_{p} \cap V_{1} \subset L^{q *} \cap V_{r}\left(q^{*}=\right.$ $d q /(d-q))$.

More precisely, there exists a positive constant $C_{q, r}$ such that

$$
\|v\|_{q^{*}}^{q}+\|\nabla v\|_{r}^{r} \leq C_{q, r}\left(\|D(v)\|_{p}^{p}+\|D(v)\|_{1}\right), \quad v \in V_{p} \cap V_{1} .
$$

(iii) If $\Omega$ is smooth and $p \geq d /(d-1)$, then $\left.v\right|_{\Omega} \in W_{0}^{1, p}(\Omega)^{d}$ for all $v \in$ $V_{p} \cap V_{1}$ where $W_{0}^{1, p}(\Omega)$ denotes the set of functions belonging to the usual Sobolev space $W^{1, p}(\Omega)$ such that $\left.\cdot\right|_{\partial \Omega}=0$.

Proof. The assertion (i) is an easy consequence of Proposition 1.1. The use of interpolation inequality;

$$
\begin{gathered}
\|f\|_{\nu} \leq\|f\|_{\lambda}^{\alpha}\|f\|_{\mu}^{\beta} \quad(1 \leq \lambda \leq \nu \leq \mu<\infty) \\
\text { with } \beta=\frac{1-\lambda / \nu}{1-\lambda / \mu} \text { and } \alpha+\beta=1
\end{gathered}
$$

and the Young inequality:

$$
A^{\alpha} B^{\beta} \leq \alpha A+\beta B \text { for } A, B \geq 0
$$

lead to

$$
\|D(v)\|_{r}^{r} \leq \frac{r-1}{p-1}\|D(v)\|_{p}^{p}+\frac{p-r}{p-1}\|D(v)\|_{1}, \quad v \in C_{0}^{\infty}\left(\mathbf{R}^{d}\right)
$$

for $1 \leq r \leq p$. Making use of (1.1) and (1.2), and keeping in mind (i) we obtain (1.9).

To prove (iii) we assume $v \in V_{p} \cap V_{1}$ and $p \geq d /(d-1)$. Then, (1.9) implies $v \in W^{1, p}\left(\mathbf{R}^{d}\right)^{d}$. Observing that $v=0$ outside of $\bar{\Omega}$ and that $\Omega$ is smooth, we obtain $\left.v\right|_{\partial \Omega}=0$.

Q. E. D.

Lemma 1.2. (i) Suppose $p \in[2, d+2)$ and let us set $q=d p /(d+2)$.

Then, we have

$$
\|\phi\|_{p}^{p} \leq\|\phi\|^{p-q}\|\phi\|_{q^{*}}^{q}, \quad \phi \in C_{0}^{\infty}\left(\mathbf{R}^{d}\right)
$$


(ii) Suppose $p \in(2 d /(d+2), 2) \cup[d+2, \infty)$. Then, there exist positive constants $K, \Lambda$ and $\theta \in(0,1)$ such that

$$
\|\phi\|_{p, B_{1 / \lambda}} \leq K \lambda^{-\theta}\left(\|\nabla \phi\|_{p}+\|\phi\|\right), \quad \phi \in C_{0}^{\infty}\left(\mathbf{R}^{d}\right)
$$

for all $\lambda \in(0, \Lambda)$, where $\|\phi\|_{p, M}=\left(\int_{M}|\phi|^{p} d x\right)^{1 / p}$.

Proof. Observing $q^{*} \geq p$ and applying (1.10) to $f=\phi$, we readily get (i). To prove (ii) we first assume $p \geq d+2$. Choose $r$ so that $r^{*}>p>d>r>1$ and set

$$
\eta_{n}(x)=\eta\left(2^{1-n} \lambda x\right), \quad n=1,2, \ldots
$$

Then, by virtue of (1.10) we have

$$
\left\|\eta_{n} \phi\right\|_{p} \leq\left\|\eta_{n} \phi\right\|^{\alpha}\left\|\eta_{n} \phi\right\|_{r^{*}}^{\beta}, \quad \beta=(p-2) r^{*} / p\left(r^{*}-2\right) .
$$

Hence, Hölder's inequality yields

$$
\left\|\eta_{n} \phi\right\|_{p} \leq C\left(\frac{2^{n}}{\lambda}\right)^{d \beta(1 / r-1 / p)}\left\|\nabla\left(\eta_{n} \phi\right)\right\|_{p}^{\beta}
$$

for all $\phi \in C_{0}^{\infty}\left(\mathbf{R}^{d}\right)$ with $\|\phi\|=1$. Choosing again $r$ so close to $d$ that

$$
0<\theta=d \beta(1 / r-1 / p)<1,
$$

we obtain from (1.13) that

$$
\begin{aligned}
\left\|\eta_{n} \phi\right\|_{p} & \leq C\left(\frac{2^{n}}{\lambda}\right)^{\theta}\left(\left\|\nabla\left(\eta_{n} \phi\right)\right\|_{p}+1\right) \\
& \leq C_{1} \lambda^{1-\theta}\|\phi\|_{p, B_{n}}+C_{2}\left(\frac{2^{n}}{\lambda}\right)^{\theta}\left(\|\nabla \phi\|_{p}+1\right),
\end{aligned}
$$

where $B_{n}=\left\{x ;|x|<2^{n} / \lambda\right\}$ and $C_{\imath}(i=1,2)$ are positive constants not depending on $\lambda$ and $n$.

Set

$$
a_{n}=\|\phi\|_{p, B_{n}}, \quad \delta=C_{1} \lambda^{1-\theta} \text { and } M=C_{2} \lambda^{-\theta}\left(\|\nabla \phi\|_{p}+1\right) .
$$

Then, (1.14) becomes $a_{n-1} \leq \delta a_{n}+2^{n \theta} M$, and hence

$$
a_{0} \leq \delta^{n} a_{n}+2^{\theta} M\left(1-2^{\theta} \delta\right)^{-1} \leq \delta^{n} a_{n}+4 M
$$

for $\lambda<\left(4 C_{1}\right)^{1 /(\theta-1)}=\Lambda$. By passage to limit we get $a_{0} \leq 4 M$. This concludes (1.12), provided $K=4 C_{2}$. 
We now suppose $2 d /(d+2)<p<2$. By virtue of Hölder's inequality we have

$$
\|\phi\|_{p, B_{1 / \lambda}} \leq \lambda^{-\theta}\|\phi\|, \quad \theta=d(1 / p-1 / 2) .
$$

Our hypothesis implies $0<\theta<1$.

Q. E. D.

Given $T>0$ and a separable Banach space $X$ equipped with norm $\|\cdot\|_{X}$, let us denote by $L^{r}(0, T ; X)(1 \leq r<\infty)$ the set of all functions $u(t)$ of the interval $(0, T)$ into $X$ such that $\|u(t)\|_{X}^{r}$ is integrable over $(0, T)$. It then follows from theorem due to Pettis and Bochner (see Yosida [18]) that there exists a sequence of finitely valued functions $u_{n}(t)$ such that $u_{n}(t) \rightarrow u(t)$ for a.e. $t \in(0, T)$ in $X$ and $u_{n} \rightarrow u$ in $L^{r}(0, T ; X)$. By $L^{\infty}(0, T ; X)$ we denote the set of all functions $u(t)$ such that $\|u(t)\|_{X}$ is essentially bounded in $(0, T)$. We use the abbreviation:

$$
L_{\text {loc }}^{r}(0, \infty ; X)=\bigcup_{T>0} L^{r}(0, T ; X) \quad(1 \leq r \leq \infty),
$$

which is a Fréchet space. By $C(I ; X)$ (resp. $C_{w}(I ; X)$ ) we denote the set of continuous functions (resp. weakly continuous functions) of $I$ into $X$.

It is not difficult to show that the space $L^{p}\left(0, T ; V_{q}\right)(p, q \geq 1)$ is separable and its dual is equal to $L^{p^{\prime}}\left(0, T ; V_{q}^{\prime}\right)\left(1^{\prime}=\infty\right)$, and hence it is reflexive if $p, q>1$.

For $a, b$ such that $0 \leq a<b$ we set

$$
\mathscr{B}_{a, b}^{p}=L^{p}\left(a, b ; V_{p}\right) \cap L^{1}\left(a, b ; V_{1}\right) . p>1,
$$

which is Banach space equipped with norm

$$
\|v\|_{a, b}=\left(\int_{a}^{b}\|v\|_{V_{p}}^{p} d t\right)^{1 / p}+\int_{a}^{b}\|v\|_{V_{1}} d t
$$

Here $L^{r}(a, b ; X)$ is defined with $(0, T)$ replaced by $(a, b)$. By $\langle,\rangle_{a, b}$ we denote the duality between $\mathscr{B}_{a, b}^{p}$ and its dual $\left(\mathscr{B}_{a, b}^{p}\right)^{\prime}$. Then, we can prove

Lemma 1.3. The space $C_{0}^{\infty}\left(0, T ; V_{p} \cap V_{1}\right)$ is dense in $\mathscr{B}_{0, T}^{p}$.

Proof. Let $u \in \mathscr{B}_{0, T}^{p}$. Since $V_{p}$ and $V_{1}$ are separable, we can find a sequence of finitely valued functions $u_{n}(t)$ such that $u_{n}(t) \rightarrow u(t)$ for a.e. $t \in(0, T)$ in $V_{p} \cap V_{1}$ and $u_{n} \rightarrow u$ in $\mathscr{B}_{0, T}^{p}$. Based on this fact, we may define the Bochner integral 


$$
u_{\varepsilon}(t)=\rho_{\varepsilon} * u(t)=\int_{0}^{T} \rho_{\varepsilon}(s) u(t-s) d s, \quad t \in(\varepsilon, T-\varepsilon)
$$

and prove that $u_{\varepsilon}$ belongs to $C^{\infty}\left(\varepsilon, T-\varepsilon ; V_{p} \cap V_{1}\right)$ and converges to $u$ in $\mathscr{B}_{\delta, T-\delta}^{p}$ as $\varepsilon \rightarrow 0$ for all $\delta \in(0, T / 2)$, where $\rho_{\varepsilon}(t)=\varepsilon^{-d} \rho(t / \varepsilon)$ (for $\rho(t)$ see (1.3)).

Let $\zeta_{\delta} \in C_{0}^{\infty}(0, T)$ be a function such that $0 \leq \zeta_{\delta}(t) \leq 1$ for all $t$ and $\zeta_{\delta}(t)=1$ for $t \in(\delta, T-\delta)$. It then easily follows that $\zeta_{\delta} u_{\varepsilon} \rightarrow \zeta_{\delta} u$ as $\varepsilon \rightarrow 0$ and $\zeta_{\delta} u \rightarrow u$ as $\delta \rightarrow 0$ in $\mathscr{B}_{0, T}^{p}$. This concludes the lemma.

Q. E. D.

Lemma 1.4. Let $u \in \mathscr{B}_{0, T}^{p}$ with $u^{\prime}=d u / d t \in\left(\mathscr{B}_{0, T}^{p}\right)^{\prime}$, which always means that

$$
\left\langle u^{\prime}, \phi\right\rangle_{0, T}=-\int_{0}^{T}\left\langle u, \phi^{\prime}\right\rangle d t, \phi \in C_{0}^{\infty}\left(0, T ; V_{p} \cap V_{1}\right) .
$$

If $p \geq 2$, we then have, after a possible modification of the value $u(t)$ on a set of measure zero,

$$
\|u(t)\|^{2}-\|u(s)\|^{2}=2\left\langle u^{\prime}, u\right\rangle_{s, t} \text { for all } 0 \leq s<t \leq T .
$$

If we further suppose $u \in C_{w}([0, T] ; H)$, then $u \in C([0, T] ; H)$.

Proof. The space $L^{\infty}\left(0, T ; V_{p} \cap V_{1}\right)$ is dense in $L^{2}(0, T ; H)$ and hence so is $\mathscr{B}_{0, T}^{p}$ if $p \geq 2$. Observing the injection $\mathscr{B}_{0, T}^{p} \rightarrow L^{2}(0, T ; H)$ is one to one and continuous, we have

$$
\mathscr{B}_{0, T}^{p} \subset L^{2}(0, T ; H) \subset\left(\mathscr{B}_{0, T}^{p}\right)^{\prime},
$$

if $p \geq 2$, where the injection $L^{2}(0, T ; H) \rightarrow\left(\mathscr{B}_{0, T}^{p}\right)^{\prime}$ is also one to one and continuous. The proof of the lemma will be thus achieved by a similar argument as in Temam [17, p. 260]. Defining $u_{\varepsilon}$ by (1.17), we have

$$
\int_{0}^{T}\left\langle u_{\varepsilon}^{\prime}, \phi\right\rangle d t=\left\langle u^{\prime}, \rho_{\varepsilon} * \phi\right\rangle_{0, T} \leq C\left\|\rho_{\varepsilon} * \phi\right\|_{0, T} \leq C\|\phi\|_{0, T}
$$

and on the other hand

$$
\int_{0}^{T}\left\langle u_{\varepsilon}^{\prime}, \phi\right\rangle d t=-\int_{0}^{T}\left\langle u_{\varepsilon}, \phi^{\prime}\right\rangle d t \rightarrow\left\langle u^{\prime}, \phi\right\rangle_{0, T} \quad \text { as } \varepsilon \rightarrow 0
$$

for all $\phi \in C^{\infty}\left(0, T ; V_{p} \cap V_{1}\right)$ with supp $\phi \subset(\varepsilon, T-\varepsilon)$. By virtue of Lemma 1.3 , we can conclude that $\left\{u_{\varepsilon}^{\prime}\right\}$ is bounded in $\left(\mathscr{B}_{0, T}^{p}\right)^{\prime}$ and that

$$
u_{\varepsilon} \rightarrow u \quad \text { in } \quad \mathscr{B}_{\delta, T-\delta}^{p},
$$




$$
u_{\varepsilon}^{\prime} \rightarrow u^{\prime} \quad \text { weakly }^{*} \text { in } \quad\left(\mathscr{B}_{\delta, T-\delta}^{p}\right)^{\prime}
$$

as $\varepsilon \rightarrow 0$, for all $\delta \in[0, T / 2)$.

According to (1.20), we have

$$
\left\|u_{\varepsilon}(t)\right\| \rightarrow\|u(t)\| \quad \text { in } L_{\mathrm{loc}}^{1}(0, T) .
$$

Hence, we can extract a subsequence, again denoted by $\left\{u_{\varepsilon}\right\}$, of $\left\{u_{\varepsilon}\right\}$ so that

$$
\left\|u_{\varepsilon}(t)\right\| \rightarrow\|u(t)\| \quad \text { as } \quad \varepsilon \rightarrow 0 \text { for all } t \in(0, T) \backslash E \text {, }
$$

where $E$ is a subset of $(0, T)$ of measure zero.

Let $s, t \in(0, T) \backslash E$ and $s<t$. Integration of the equality

$$
\frac{d}{d \tau}\left\|u_{\varepsilon}(\tau)\right\|^{2}=2\left\langle u_{\varepsilon}^{\prime}(\tau), u_{\varepsilon}(\tau)\right\rangle
$$

over $(s, t)$ leads to

$$
\left\|u_{\varepsilon}(t)\right\|^{2}-\left\|u_{\varepsilon}(s)\right\|^{2}=2\left\langle u_{\varepsilon}^{\prime}, u_{\varepsilon}\right\rangle_{s, t} .
$$

Letting $\varepsilon \rightarrow 0$ here, we easily see (1.19), keeping in mind (1.20) (1.22). Since the right-hand side of (1.19) is continuous in $s$ and $t$, we get (1.19) for all $0 \leq s<t \leq T$, modifying, if necessary, the value of $u(t)$ on $E$. The latter half of the lemma easily follows from the continuity of $\|u(t)\|$ Q. E. D.

Finally, we describe a few statements about functional $\varphi$ and operator $B$. Regarding the properties which are maintained by the functional $(0.3)$, we are going to introduce a class of functionals on $V_{p}$. For each $t \geq 0$ we consider a functional $\varphi_{t}(u)=\varphi(t, u)$ on $V_{p}, p \geq 1$, possessing the properties (A.1) (A.3):

(A.1) For each $t \geq 0 \varphi_{t}$ is a proper, convex and lower-semicontinuous function on $V_{p}$ such that $\varphi_{t}(0)=0$.

(A.2) There exist positive constants $\mu_{i}$ and $g_{i}(i=1,2)$ such that for all $t \geq 0$ and all $v \in W_{p}$

$\varphi_{t}(u) \geq \mu_{1}\|D(u)\|_{p}^{p}+g_{1}\|D(u)\|_{1}, \quad u \in V_{p} \cap V_{1}$,

$\left|\left\langle\partial \varphi_{t}(u), v\right\rangle\right| \leq \mu_{2} \int_{\Omega}|D(u)|^{p-1}|D(v)| d x+g_{2}\|D(v)\|_{1}, u \in \mathscr{D}\left(\partial \varphi_{t}\right)$,

where $\partial \varphi_{t}(u)$ denotes the set of subgradients of $\varphi$ at $u$ :

$$
\partial \varphi_{t}(u)=\left\{w \in W_{p}^{\prime} ; \varphi_{t}(v)-\varphi_{t}(u) \geq\langle w, v-u\rangle, \quad v \in W_{p}\right\},
$$


$\mathscr{D}\left(\partial \varphi_{t}\right)$ the effective domain of $\partial \varphi_{t}$ :

$$
\mathscr{D}\left(\partial \varphi_{t}\right)=\left\{u \in W_{p} ; \partial \varphi_{t}(u) \neq \phi\right\},
$$

and hence $\partial \varphi_{t}$ may be regarded as a mapping of $\mathscr{D}\left(\partial \varphi_{t}\right)$ into the set of subsets of $W_{p}^{\prime}$.

(A.3) There exists a positive constant $\varepsilon(h)$ depending on $h \geq 0$ such that $\varepsilon(h) \rightarrow 0$ as $h \rightarrow 0$, and for all $s, t \geq 0$ and all $v \in V_{p} \cap V_{1}$

$$
|\varphi(s, v)-\varphi(t, v)| \leq \varepsilon(|s-t|)\left(\|D(v)\|_{p}^{p}+\|D(v)\|_{1}\right) .
$$

It may be easily shown that $0 \in \mathscr{D}\left(\partial \varphi_{t}\right) \subset W_{p} \cap V_{1}$ and

$$
\varphi_{t}(u) \leq \mu_{2}\|D(u)\|_{p}^{p}++g_{2}\|D(u)\|_{1}, \quad u \in \mathscr{D}\left(\partial \varphi_{t}\right) .
$$

For a future convenience we set

$$
\Phi_{p}=\text { the set of } \varphi_{t}, t \geq 0 \text {, satisfying (A.1) (A.3). }
$$

It is well-known (see Brezis [3]) that $\varphi(t, v(t))$ is measurable function of $t \geq 0$ if $v \in L^{p}\left(0, T ; V_{p}\right)$ and a mapping $v \rightarrow \int_{0}^{T} \varphi(t, v(t)) d t$ is convex and lower-semicontinuous.

Finally, we describe two lemmas concerning operator $B(u)=u \cdot \nabla u$.

Lemma 1.5. Suppose $d=3$. For each $p>6 / 5$ there exists a positive constant $\gamma_{p}$ such that

$$
\left|\left\langle u_{1} \cdot \nabla u_{2}, v\right\rangle\right| \leq \gamma_{p}\left(\left\|u_{1}\right\|\left\|u_{2}\right\|\right)^{a / 2}\left(\left\|\nabla u_{1}\right\|_{l}\left\|\nabla u_{2}\right\|_{l}\right)^{b / 2}\|\nabla v\|_{q}
$$
for all $u_{1}, u_{2}, v$ in $\mathscr{V}$, where $a+b=2$ and

$$
\begin{aligned}
& b=p-1, \quad l=p, \quad q=\frac{6 p}{(5 p-6)(p-1)} \text { when } 6 / 5<p<11 / 5, \\
& b=\frac{6}{5 p-6}, l=p, \quad q=p \quad \text { when } 9 / 5 \leq p<3 \text {, } \\
& b=1, \quad l=\frac{6 p}{5 p-6}, q=p \quad \text { when } 12 / 5 \leq p<\infty \text {. }
\end{aligned}
$$

When $d=2$, the inequality $(1.25)$ is valid for all $p>1$, provided that

$$
\begin{array}{llll}
b=p-1, & l=p, & q=\frac{p}{(p-1)^{2}} & \text { when } 1<p<2, \\
b=1, & l=p^{\prime}, & q=p & \text { when } 2 \leq p<\infty,
\end{array}
$$


where $p^{\prime}=p /(p-1)$.

Proof. We start with case $d=3$.

(i ) Let $p \in(6 / 5,11 / 5)$. By integration by part we have, using Hölder's inequality,

$$
\left|\left\langle u_{1} \cdot \nabla u_{2}, v\right\rangle\right| \leq C\left\|u_{1}\right\|_{2 q^{\prime}}\left\|u_{2}\right\|_{2 q^{\prime}}\|\nabla v\|_{q}, q^{\prime}=q /(q-1) .
$$

Applying (1.10) with $\lambda=2, \mu=p^{*}=3 p /(3-p)$ and $\nu=2 q^{\prime}$, we get, using (1.2),

$$
\left\|u_{i}\right\|_{2 q^{\prime}} \leq C\left\|u_{i}\right\|^{a / 2}\left\|\nabla u_{i}\right\|_{p}^{b / 2}, \quad i=1,2
$$

Substituting these into (1.26) leads to (1.25).

(ii) Let $p \in[9 / 5,3)$. Take $q=p$ in (1.26). Keeping in mind that $2<2 p^{\prime}$ $\leq p^{*}$, we obtain analogously as in (i)

$$
\left\|u_{i}\right\|_{2 p^{\prime}} \leq C\left\|u_{i}\right\|^{\alpha}\left\|\nabla u_{i}\right\|_{p}^{\beta}
$$

where $\alpha+\beta=1$ and $\beta=3 /(5 p-6)$. Combining this with $(1.26)(q=p)$, we arrive at (1.25).

(iii) Let $p \in[12 / 5, \infty)$. Since $2<2 p^{\prime}<r=2 p /(p-2)$ and $1 / r=1 / l$ $-1 / 3$, we have

$$
\left\|u_{i}\right\|_{2 p^{\prime}} \leq C\left\|u_{i}\right\|^{1 / 2}\left\|\nabla u_{i}\right\|_{l}^{1 / 2} .
$$

Inserting this into (1.26) with $q=p$ leads to (1.25).

Exactly as above we can show (1.25) for the case $d=2$.

Q. E. D.

The following lemma is an immediate consequence of Proposition 1.1 and the previous lemma.

Lemma 1.6. Suppose that $d=3$ and $u \in \mathscr{B}_{0, T}^{\mathrm{p}} \cap L^{\infty}(0, T ; H)$. Then, $B(u)=$ $u \cdot \nabla u$ is contained in $L^{r^{\prime}}\left(0, T ; V_{q}^{\prime}\right)$, where

$$
\begin{gathered}
r=p, \quad q=q(p)= \begin{cases}6 p /\{(5 p-6)(p-1)\}, & p \in(6 / 5,11 / 5) \\
p, & p \in[11 / 5, \infty)\end{cases} \\
\left(\text { or } r^{\prime}=p(5 p-6) / 6, \quad q=p, \quad p \in[9 / 5,11 / 5)\right) .
\end{gathered}
$$

\section{§2. Results and remarks}

Theorem 1 (Existence of weak solutions). Suppose that $\Omega$ is a domain in $\mathbf{R}^{3}$, 
that $\varphi_{t}$ is contained in the set $\Phi_{p}, p>6 / 5$, which appears in (1.24), and that the prescribed data $u_{0}$ and $f$ satisfy

$$
u_{0} \in H \quad \text { and } \quad f \in L_{\mathrm{loc}}^{2}(0, \infty ; H) .
$$

There then exists a weak solution, i.e., a vector field $u$ satisfying

$$
u \in \underset{T>0}{\cup} \mathscr{B}_{0, T}^{p} \cap C_{w}([0, T] ; H) \quad\left(\mathscr{B}_{0, T}^{p}=L^{p}\left(0, T ; V_{p}\right) \cap L^{1}\left(0, T ; V_{1}\right)\right)
$$

with a derivative $u^{\prime}(t)=d u(t) / d t$ :

$$
u^{\prime} \in\left\{\underset{T>0}{\cup} \mathscr{B}_{0, T}^{p} \cap L^{p}\left(0, T ; V_{q}\right)\right\}^{\prime} \quad \text { in the sense (1.18), }
$$

the initial condition

$$
u(0)=u_{0}
$$

the evolutional inequality

$$
\begin{aligned}
& \int_{0}^{T}\left\langle v^{\prime}, v-u\right\rangle d t-\frac{1}{2}\left(\|v(T)-u(T)\|^{2}-\left\|v(0)-u_{0}\right\|^{2}\right) \\
& \quad+\int_{0}^{T}\langle B(u), v\rangle d t+\int_{0}^{T}\{\varphi(t, v)-\varphi(t, u)\} d t \geq \int_{0}^{T}\langle f, v-u\rangle d t
\end{aligned}
$$

for all $T>0$ and all $v \in W_{0, T}^{p}$ :

$$
W_{0, T}^{p}=\left\{v \in \mathscr{B}_{0, T}^{p} \cap L^{p}\left(0, T ; V_{q}\right) \cap C_{w}([0, T] ; H) ; v^{\prime} \in\left(\mathscr{B}_{0, T}^{p}\right)^{\prime}\right\}
$$

and the energy inequality

$$
\frac{1}{2}\|u(t)\|^{2}+\int_{0}^{t} \varphi(\tau, u) d \tau \leq \frac{1}{2}\left\|u_{0}\right\|^{2}+\int_{0}^{t}\langle f, u\rangle d \tau \text { for all } t>0
$$

where $q=q(p)$ is the same as in (1.27). In particular,

$$
u \in L^{p}(\Omega \times(0, T)) \text { for any } T>0 \text { when } 2 \leq p<5 .
$$

COROLlary 1 (Existence of strong solutions). Suppose $p \geq 2$ in Theorem 1 and let $u$ be a weak solution satisfying

$$
u \in L_{\mathrm{loc}}^{q}\left(0, \infty ; V_{p}\right) \text { with } q=q(p) \text { from }(1.27) .
$$

Then, it is a strong solution, i.e., a weak solution possessing the further properties:

$$
\text { (i) } u \in C([0, T] ; H), \quad \text { (ii) } u^{\prime} \in\left(\underset{T>0}{\cup} \mathscr{B}_{0, T}^{p}\right)^{\prime} \text {, }
$$




$$
\begin{aligned}
&\left\langle u^{\prime}, v-u\right\rangle_{0, T}+\int_{0}^{T}\langle B(u), v-u\rangle d t+\int_{0}^{T}\{\varphi(t, v)-\varphi(t, u)\} d t \\
& \geq \int_{0}^{T}\langle f, v-u\rangle d t \quad \text { for all } T>0 \text { and all } v \in \mathscr{B}_{0, T}^{p}
\end{aligned}
$$

and the energy inequality of strong form

$$
\frac{1}{2}\|u(t)\|^{2}+\int_{s}^{t} \varphi(\tau, u) d \tau \leq \frac{1}{2}\|u(s)\|^{2}+\int_{s}^{t}\langle f, u\rangle d \tau
$$

for all $0 \leq s<t$, where $\langle,\rangle_{0, T}$ denotes the duality between $\mathscr{B}_{0, T}^{p}$ and its dual. Particularly, if $p \geq 11 / 5$, there then exists a strong solution.

Proof. If $p \geq 11 / 5$, then (2.3) implies (ii) of (2.10). Suppose $p<11 / 5$. Application of (1.25) yields

$$
\int_{0}^{T}\|B(u)\|_{V_{p^{\prime}}}^{p^{\prime}} d t \leq \gamma_{p} \sup _{0 \leq t \leq T}\|u(t)\|^{a p^{\prime}} \int_{0}^{T}\|\nabla u\|_{p}^{b p^{\prime}} d t,
$$

from which (ii) of (2.10) follows (see (4.3)). Here, $b=6 /(5 p-6)$ and $p^{\prime}=p /(p-1)$. Then, (i) of $(2.10)$ is an easy consequence of Lemma 1.4.

For any $v \in C^{1}\left([0, T] ; V_{p} \cap V_{1}\right)$ it follows from Lemma 1.4 that

$$
\begin{aligned}
\int_{0}^{T}\left\langle v^{\prime}, v-\right. & u\rangle d t \leq\left\langle u^{\prime}, v-u\right\rangle_{0, T} \\
& +\frac{1}{2}\left(\|u(T)-v(T)\|^{2}-\left\|u_{0}-v(0)\right\|^{2}\right)
\end{aligned}
$$

and hence we have (2.11) for such $v$. Let $v \in \mathscr{B}_{0, T}^{p}$. We make an extension of $v(t)$ so that $v(t)=0$ for $t<0$ and for $t>T$, and define a mollifier

$$
v_{\varepsilon}(t)=\int_{-\infty}^{\infty} \rho_{\varepsilon}(s) v(t-s) d s
$$

which belongs to $C^{1}\left([0, T] ; V_{p} \cap V_{1}\right)$ and converges to $v$ in $\mathscr{B}_{0, T}^{p}$ as $\varepsilon \rightarrow 0$. Inserting $v=v_{\varepsilon}$ in (2.11) and letting $\varepsilon \rightarrow 0$, we obtain (2.11) for all $v \in \mathscr{B}_{0, T}^{p}$ and all $T>0$. In fact, since $\varphi_{t}$ is convex, we have

$$
\begin{gathered}
\varphi\left(t, v_{\varepsilon}(t)\right) \leq \int_{-\infty}^{\infty} \rho_{\varepsilon}(s) \varphi(t-s, v(t-s)) d s \\
\left.+\int_{-\infty}^{\infty} \rho_{\varepsilon}(s)\{\varphi(t, v(t-s))-\varphi(t-s), v(t-s))\right\} d s=\mathrm{I}_{\varepsilon}(t)+\mathrm{II}_{\varepsilon}(t) .
\end{gathered}
$$


Keeping in mind that $\varphi(t, v(t))$ is integrable on $(0, T)$, we get $I_{\varepsilon}(t) \rightarrow$ $\phi(t, v(t))$ in $L^{1}(0, T)$. An elementary calculation gives us

$$
\int_{0}^{T}\left|\mathrm{II}_{\varepsilon}(t)\right| d t \leq \int_{-\infty}^{\infty} \rho_{\varepsilon}(s) d s \int_{-s}^{T}|\varphi(\tau+s, v(\tau))-\varphi(\tau, v(\tau))| d \tau .
$$

Employing the Lebesgue theorem, we can derive from (A.3) that

$$
\lim _{s \rightarrow 0} \int_{-s}^{T}|\varphi(\tau+s, v(\tau))-\varphi(\tau, v(\tau))| d \tau=0,
$$

which proves $\operatorname{II}_{\varepsilon}(t) \rightarrow 0$ in $L^{1}(0, T)$ and hence (2.15) yields

$$
\limsup _{\varepsilon \rightarrow 0} \int_{0}^{T} \varphi\left(t, v_{\varepsilon}(t)\right) d \tau \leq \int_{0}^{T} \varphi(t, v(t)) d t .
$$

The inequality (2.12) is an easy consequence of (2.11) and Lemma 1.4. Q. E. D.

Corollary 2 (Uniqueness of strong solutions). Suppose in Theorem 1 that $\varphi_{t}$ is written in the form

$$
\varphi_{t}(v)=\hat{\varphi}_{t}(v)+\int_{\Omega} \mu(t)|D(v)|^{2} d x
$$

where $\hat{\varphi}_{t} \in \Phi_{r}, r \leq 1$, and $\mu \in C\left([0, \infty), L^{\infty}(\Omega)\right)$ satisfying $\mu \geq \mu_{0}$ for a positive constant $\mu_{0}>0$. Then, we have:

(i ) $\varphi_{t} \in \Phi_{p}$ with $p=\max (2, r)$.

(ii) Let $u_{*}$ be a weak solution and $u$ be a strong solution satisfying (2.10) and (2.11), and further assume that $u \in L^{2 q /(2 q-3)}\left(0, T ; V_{q}\right)$ for $q=q(p)$ from (1.27) and for all $T>0$. Then, $u=u_{*}$.

Proof. (i) If $p \geq 2$, then $|D(u)||D(v)| \leq\left(|D(u)|^{p-1}+1\right)|D(v)|$. If $p<2$, we have, using (1.11),

$$
\begin{aligned}
|D(u)|^{p-1}|D(v)| & =(|D(u)||D(v)|)^{p-1}|D(v)|^{2-p} \\
& \leq(p-1)|D(u)||D(v)|+(2-p)|D(v)| .
\end{aligned}
$$

Consequently, ( $i$ ) follows from (1.23).

(ii) It is evident that $p \geq 2$ leads to $2 q /(2 q-3) \geq p$. Therefore, we have $u \in L^{p}\left(0, T ; V_{q}\right)$ and hence it follows from ( ii ) of (2.10) that $u$ is in $W_{0, T}^{p}$ for $T>0$. We choose $v=u$ as a test function in the variational inequality (2.5) with $u$ and $T$ replaced by $u_{*}$ and $t$, and get 


$$
\begin{gathered}
\int_{0}^{t}\left\{\left\langle u^{\prime}, u-u_{*}\right\rangle+\left\langle B\left(u_{*}\right), u\right\rangle+\hat{\varphi}(\tau, u)-\hat{\varphi}\left(\tau, u_{*}\right)\right\} d \tau \\
\geq \frac{1}{2}\left\|u(t)-u_{*}(t)\right\|^{2}+\int_{0}^{t}\left\{\left\langle 2 \mu D\left(u_{*}\right), D\left(u-u_{*}\right)\right\rangle+\left\langle f, u-u_{*}\right\rangle\right\} d \tau .
\end{gathered}
$$

Inserting $v=u_{*}$ into (2.11) and adding this to (2.17), we obtain

$$
\|w(t)\|^{2}+2 \mu_{0} \int_{0}^{t}\|\nabla w\|^{2} d \tau \leq 2 \int_{0}^{t}\langle B(w), u\rangle d \tau, w=u-u^{*}
$$

from which we are going to derive $w(t)=u(t)-u_{*}(t)=0$ for every $t$. To do so, we use (1.2), (1.10) $\left(2<2 q^{\prime}<6\right)$ and (1.11) to get the following:

$$
\text { LHS of }(2.18) \leq 2 \int_{0}^{t}\|\nabla u\|_{q}\|w\|_{2 q^{\prime}}^{2} d \tau
$$

$$
\begin{gathered}
\left.\leq 2 \int_{0}^{t}\|\nabla u\|_{q}\|w\|^{2 \alpha}\|w\|_{6}^{2 \beta} d \tau \leq 2\left(\eta \int_{0}^{t}\|w\|_{6}^{2} d \tau\right)^{\beta}\left(\eta^{-\beta / \alpha} \int_{0}^{t}\|\nabla u\|_{q}^{1 / \alpha}\|w\|^{2}\right) d \tau\right)^{\alpha} \\
\leq 2 \beta \eta \int_{0}^{t}\|w\|_{6}^{2} d \tau+2 \alpha \eta^{-\beta / \alpha} \int_{0}^{t}\|\nabla u\|_{q}^{1 / \alpha}\|w\|^{2} d \tau \\
\leq 2 \mu_{0} \int_{0}^{t}\|\nabla w\|^{2} d \tau+2 \alpha \eta^{-\beta / \alpha} \int_{0}^{t}\|\nabla u\|_{q}^{1 / \alpha}\|w\|^{2} d \tau
\end{gathered}
$$

where $d=1-3 / 2 q, \beta=1-\alpha$ and $\eta=\mu_{0} / \beta S_{2}^{2}$. From this it follows that

$$
\|w(t)\|^{2} \leq C \int_{0}^{t}\|\nabla u\|_{q}^{1 / \alpha}\|w\|^{2} d \tau
$$

Keeping in mind that $\|\nabla u\|_{q}^{1 / \alpha} \in L^{1}(0, T)$, we conclude that $u(t)=u_{*}(t)$ for all $t$.

Q. E. D.

COROLlary 3 (Energy decay). Let $u$ be a weak solution which is obtained in Theorem 1. Then, the following statements hold.

(i) If $\in L^{1}(0, \infty ; H)$ and if $u$ satisfies (2.12), then $\|u(t)\| \rightarrow 0$ as $t \rightarrow \infty$.

(ii) If $f$ satisfies $\|f(t)\|_{3} \leq g_{1} / S_{1}$ for all $t \geq 0$, then $\|u(t)\| \leq\left\|u_{0}\right\|$ for all $t \geq 0$, where $S_{1}$ and $g_{1}$ are constants appearing in (1.2) and (1.23), respectively.

(iii) Assume that $u$ is a strong solution satisfying (2.9) and $u^{\prime} \in L^{r}(0, \infty$; $\left.V_{p}^{\prime} \cap L^{3}(\Omega)\right)$ for some $r \geq p^{\prime}$. If $f$ satisfies $\|f(t)\|_{3}<g_{1} / S_{1}$ for all $t \geq T_{0}$, then there exists $T_{1} \geq T_{0}$ such that $u(t)=0$ for all $t \geq T_{1}$.

Proof. (i ) From (2.12) with $s=0$ it follows by using Gronwall's lemma that 


$$
\|u(t)\|^{2}+2 \int_{0}^{t} \varphi(\tau, u) d \tau \leq \text { const. for all } t>0
$$

which implies $u \in \mathscr{B}_{0, \infty}^{p} \cap L^{\infty}(0, \infty ; H)$. Hence, $u(t) \in V_{p} \cap V_{1}$ for a.e. $t>0$. Applying (1.9) with $v=u(t)$ and $q=6 / 5$, we obtain $u \in L^{6 / 5}(0, \infty ; H)$ since $q^{*}$ $=2$. Therefore, the proof of (i) will be achieved by carrying out the same device as in Miyakawa-Sohr [11].

(ii) Using (1.2) and (1.23), we can derive from (2.7)

$$
\frac{1}{2}\|u(t)\|^{2}+\int_{0}^{t}\left\{\mu_{1}\|\nabla u\|_{p}^{p}+\left(g_{1}-S_{1}\|f\|_{3}\right)\|D(u)\|_{1}\right\} d \tau \leq \frac{1}{2}\left\|u_{0}\right\|^{2},
$$

which implies (ii).

(iii) After a simple calculation we obtain from (2.11) that

$$
\varphi(t, u(t)) \leq\left\langle f(t)-u^{\prime}(t), u(t)\right\rangle \text { for a.e. } t \geq 0 .
$$

On the other hand it easily follows from the assumption that there exists $T_{1} \geq T_{0}$ such that $\left\|u^{\prime}\left(T_{1}\right)\right\|_{3}+\left\|f\left(T_{1}\right)\right\|_{3} \leq g_{1} / S_{1}$ and $(2.21)$ is valid for $t=T_{1}$. Inserting $t=T_{1}$ into (2.21), we readily obtain $\varphi\left(T_{1}, u\left(T_{1}\right)\right) \leq g_{1}\left\|D\left(u\left(T_{1}\right)\right)\right\|_{1}$, and hence $u\left(T_{1}\right)=0$. It is easy to see that $u$ is a weak solution for $t \geq T_{1}$ with initial data $u\left(T_{1}\right)=0$. Thus, part (ii) guarantees that $u(t)=0$ for all $t \geq T_{1}$.

Q. E. D.

Theorem 2 (Case of exterior domain). Suppose that the complement of $\Omega$ is compact and that $\varphi(u)=\mu\|D(u)\|_{p}^{p}+g\|D(u)\|_{1}$ with $p \geq 9 / 5$ and positive constants $\mu, g$. Then, for any data (2.1) there exists a weak solution $u$ satisfying the energy inequality of strong form

$$
\begin{aligned}
\frac{1}{2}\|u(t)\|^{2}+\int_{s}^{t}\left\{p \mu\|D(u)\|_{p}^{p}+g\|D(u)\|_{1}\right\} d \tau & \\
& \leq \frac{1}{2}\|u(t)\|^{2}+\int_{s}^{t}\langle f, u\rangle d \tau
\end{aligned}
$$

for $s=0$, a.e. $s>0$ and all $t \geq s$.

In the last theorem we consider a Bingham fluid with variable viscosity $\mu$ and yield limit $g$, which is occupied in a bounded and smooth domain $\Omega$ in $\mathbf{R}^{3}$. We recall that $V_{p}(p \geq 3 / 2)$ is identified with the closure of $\mathscr{V}(\Omega)$ by norm $\|\nabla v\|_{p}$ (see Lemma 1.1 (iii)). Set

$$
\varphi(t, u)=\int_{\Omega}\left\{\mu(t)|D(u)|^{2}+g(t)|D(u)|\right\} d x \quad \text { for } \quad u \in V
$$


For prescribed data $u_{0}$ and $f$ :

$$
u_{0} \in V \text { and } f \in W_{\mathrm{loc}}^{1,1}(0, \infty ; H)
$$

we consider the problem: To find a strong solution satisfying the evolutional inequality

$$
\left\langle u^{\prime}(t)+B(u(t)), v-u(t)\right\rangle+\varphi(t, v)-\varphi(t, u(t)) \geq\langle f(t), v-u(t)\rangle,
$$
for $v \in V$ and for a.e. $t>0$, and the initial condition

$$
u(0)=u_{0} \text { in } \Omega .
$$

Before stating the theorem we introduce two function spaces $\mathcal{M}$ and $\mathscr{G}$ in which $\mu$ and $g$ are contained, respectively. To do so, for $b>6$ we define $a$ and $\alpha$ as follows:

$$
\frac{1}{a}+\frac{1}{b}=\frac{1}{2} \text { and } \quad \frac{1}{a}+\frac{1}{3}=\frac{1}{\alpha}+\frac{1}{2}
$$

It is obvious that $2<a<3,1 / \alpha+1 / b=1 / 3$ and hence $3<\alpha<6$. Then, we define

$$
\begin{aligned}
& \mathcal{M}=\left\{\mu \in C\left([0, \infty) ; W^{1, \alpha}(\Omega)\right) ; \mu^{\prime} \in L_{\mathrm{loc}}^{2}\left(0, \infty ; L^{b}(\Omega)\right)\right\} \\
& \mathscr{G}=W_{\mathrm{loc}}^{1,2}\left(0, \infty ; L^{2}(\Omega)\right) .
\end{aligned}
$$

Denoting by $\gamma_{0}, \gamma_{1}$ and $c_{0}$ positive constants such that

$$
|\langle B(u), v\rangle \cdot| \leq \frac{\gamma_{0}}{8}\|\nabla u\|^{2}\|v\|_{3}, \quad\|v\|_{3}^{4} \leq c_{0}\|v\|^{2}\|\nabla v\|^{2}
$$

and

$$
|\langle B(u), v\rangle| \leq \frac{1}{8}\left(\eta\|\nabla u\|^{2}+4 \gamma_{1} \eta^{-3}\|u\|^{2}\right)\|\nabla v\|, \quad \eta>0
$$

for all $u, v \in V$, and setting for all $T>0$

$$
\begin{aligned}
& A_{T}=\left(\left\|u_{0}\right\|^{2}+\int_{0}^{T}\|f\| d t\right) \exp \left(\int_{0}^{T}\|f\| d t\right), \\
& M_{T}=C \mu_{1} \mu_{0}^{-2}\left(\sup _{0 \leq t \leq T}\|\nu(t) \nabla \mu(t)\|_{\alpha}^{2}+1\right) \int_{0}^{T}\left\|\nu \mu^{\prime}\right\|_{b}^{2} d t, \\
& G_{T}=\int_{0}^{T}\left\|\sqrt{\nu} g^{\prime}\right\|^{2} d t,
\end{aligned}
$$




$$
\begin{gathered}
I_{T}=\left\{\|f(0)-\chi\|^{2}+\int_{0}^{T}\left\|f^{\prime}\right\| d t+\underset{0 \leq t \leq T}{\left.\left(\max _{0}\|f(t)\|^{2}+g_{1}^{2}\right) M_{T}+G_{T}\right\}}\right. \\
\quad \times \exp \left(\int_{0}^{T}\left\|f^{\prime}\right\| d t+\gamma_{1} \mu_{0}+M_{T}\right), \\
J_{T}=M_{T} \exp \left(\int_{0}^{T}\left\|f^{\prime}\right\| d t+\gamma_{1} \mu_{0}+M_{T}\right)
\end{gathered}
$$

and

$$
E_{T}=\left(18 \mu_{0}^{\lambda-2} A_{T}^{1+\lambda} J_{T}\right)^{1 / \lambda}+18 \mu_{0} A_{T} J_{T}+\left\{18 A_{T}\left(\max _{0 \leq t \leq T}\|f(t)\|^{2}+I_{T}\right)\right\}^{1 / 2}
$$

with $\nu=1 / \mu, \lambda=3 / \alpha-1 / 2$, positive constants $\mu_{i}(i=0,1)$ and some positive constant $C$ depending only on $\alpha$ and $\Omega$, we can state the last theorem.

THEOREM 3. Let $\Omega$ be a bounded and smooth domain in $\mathbf{R}^{3}$ and let $\mu_{i}$, $g_{i}(i=0,1)$ be positive constants. Suppose that $\mu \in \mathcal{M}, g \in \mathscr{G}, \mu_{0} \leq \mu \leq \mu_{1}$ and $g_{0} \leq g \leq g_{1}$, and that $u_{0}$ and $f$ satisfy (2.24) and

$$
\chi-B\left(u_{0}\right) \in \partial \varphi\left(0, u_{0}\right) \text { for some } \chi \in H .
$$

If one of the following conditions

$$
\text { (i ) } \mu_{0}^{5} / \gamma_{0}^{4}>c_{0} A_{T} E_{T} \text { with } \gamma_{1}=0 \text { and (ii) } \mu_{0}^{3}>T^{1 / 2} E_{T}
$$

is fulfilled, then we can find a strong solution $u$ satisfying (2.25), (2.26) and

$$
\begin{aligned}
& \mu_{0}\|\nabla u(t)\|^{2} \leq E_{T}, \\
& \left\|u^{\prime}(t)\right\|^{2}+\frac{\mu_{0}}{4} \int_{0}^{T}\left\|\nabla u^{\prime}\right\|^{2} d t \leq I_{T}+J_{T}\left(\mu_{0} E_{T}+\mu_{0}^{\lambda-2} A_{T}^{\lambda} E_{T}^{2-\lambda}\right)
\end{aligned}
$$

for all $t \leq T$. Moreover, the $u$ is unique in the sense that every weak solution is equal to $u$. In particular, if $f$ is in $L_{\mathrm{loc}}^{\infty}\left(0, \infty ; L^{3}(\Omega)^{3}\right)$, the following

$$
\begin{aligned}
& \sup _{0 \leq t \leq T}\|\nabla u(t)\|_{q}(2 \leq q \leq 6) \text { and } \\
& \int_{0}^{T}\|\nabla u\|_{q}^{p} d t\left(q>6, \frac{1}{p}=\frac{1}{4}\left(1-\frac{6}{q}\right)\right)
\end{aligned}
$$

are bounded from above by positive continuous functions of the arguments

$$
\begin{gathered}
\|\chi\|, \mu_{0}, \mu_{1}, g_{1}, \int_{0}^{T}\left(\|f\|+\left\|f^{\prime}\right\|\right) d t, \\
\sup _{0 \leq t \leq T}\|\nu \nabla u(t)\|_{\alpha}, \int_{0}^{T}\left\|\nu \mu^{\prime}\right\|_{b}^{2} d t, \int_{0}^{T}\left\|\sqrt{\nu} g^{\prime}\right\|^{2} d t .
\end{gathered}
$$


Remark 1. Suppose $d=2$. Reviewing Lemma 1.5 and the procedure carried out in Section 3, we obtain a new version of Theorem 1: Let $p>1$. For any data (2.1) there exists a weak solution $u(t)$ satisfying (2.2) (2.7) for all $T>0$ and all $v \in W_{0, T}^{p}$, where $q=p /(p-1)^{2}$ if $1<p<2$ and $q=p$ if $p \geq 2$. Accordingly, it follows from Corollaries 1 and 2 , by taking $q=p$ and applying the inequality $\|w\|_{2 p}$, $\leq$ const. $\|w\|^{1 / p^{\prime}}\|\nabla w\|^{1 / p}$ in the place of (2.19), that there exists exactly one strong solution if $p \geq 2$ and $\varphi_{t}$ is written in the form (2.16).

Remark 2. The conclusion of Theorem 2 remains valid even if $\varphi(u)$ is replaced by

$$
\sum_{j=1}^{N} \mu_{j}\|D(u)\|_{p_{j}}^{p_{j}} \text { with } \max \left(p_{j}\right) \geq 9 / 5 \quad \text { and } \min \left(p_{j}\right)=1 .
$$

Remark 3. Let $\varphi$ be a functional not depending on $t$ and satisfying (A.1) (A.2) for $p>6 / 5$, provided $W_{p}$ is replaced by $V_{p} \cap V_{9 / 5}$. Then, it is easily shown that for any $f \in H$ there exists a solution $u \in V_{p} \cap V_{1}$ to the stationary problem:

$$
\langle B(u), v\rangle+\varphi(v)-\varphi(u) \geq\langle f, v-u\rangle, \quad v \in V_{q} \cap V_{1},
$$

where $q=3 p /(5 p-6)$ for $p \in(6 / 5,9 / 5)$ and $q=p$ for $p \geq 9 / 5$. In fact, observing (1.26) with $2 q^{\prime}=p^{*}(6 / 5<p<9 / 5)$ and (1.9) $(q=6 / 5)$, we can find $u_{\xi} \in \mathscr{D}(\partial \varphi) \subset V_{p} \cap V_{9 / 5}$ satisfying $f \in B\left(u_{\xi}\right)+e_{\xi}\left(u_{\xi}\right)+\partial \varphi\left(u_{\xi}\right)$ as in Proposition 3.1, where $e_{\xi}(v)=-\xi \nabla\left(|\nabla v|^{-1 / 5} \nabla v\right)$ and $\xi$ is a positive constant. A desired solution $u$ is given as a limit of $u_{\xi}$ (cf. Lemma 1.5).

Remark 4. Suppose $d=2$. For any $b>2$ we define $a$ and $\alpha$ by $1 / a+1 / b$ $=1 / 2$ and $\alpha=a>2$. Then, Theorem 3 remains valid without condition (2.31). More precisely, under the same hypotheses as in Theorem 3 we can prove that if $u_{0}$ and $f$ satisfy (2.30), then there exists one and only one solution of (2.25)-(2.26) in $t \leq T$ satisfying

$u \in L^{\infty}\left(0, T ; V_{q}\right)$ for any $q \geq 2$, and $u^{\prime} \in L^{2}(0, T ; V) \cap L^{\infty}(0, T ; H)$.

\section{§3. Regularized problem}

For positive numbers $\lambda$ and $\xi$ we define an operator $e_{\lambda, \xi}$ of $V=V_{2}$ into its dual $V^{\prime}$ by

$$
\left\langle e_{\lambda, \xi}(u), v\right\rangle=\xi\left\langle\exp \left(\lambda\|\nabla u\|^{c}\right) \nabla u, \nabla v\right\rangle \text { for all } v \in V \text { with } c>4
$$


It is easy to see that $e_{\lambda, \xi}$ is monotone and $B\left(u_{n}\right)=u_{n} \cdot \nabla u_{n} \rightarrow u \cdot \nabla u$ weakly in $V^{\prime}$ if $u_{n} \rightarrow u$ weakly in $V$. Accordingly, $A=e_{\lambda, \xi}+B: u \rightarrow e_{\lambda, \xi}(u)+B(u)$ is a pseudo-monotone operator of $V$ into $V^{\prime}$, i.e., if $\|u\|_{V} \leq 1$, then $\|A(u)\|_{V}$, is bounded, and if $u_{j} \rightarrow u$ weakly in $V$ as $j \rightarrow \infty$ and $\lim \sup \left\langle A\left(u_{j}\right), u_{j}-u\right\rangle \leq 0$, then $\liminf _{j \rightarrow \infty}\left\langle A\left(u_{j}\right), u_{j}-v\right\rangle \geq\langle A(u), u-v\rangle$ for all $v \in V$. It is readily seen that the

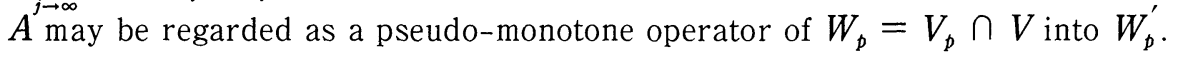

Proposition 3.1. Let $\varphi \in \Phi_{p}, p>6 / 5$, which does not depend on $t$, let $L_{\lambda, \xi}$ be a mapping from $\mathscr{D}(\partial \varphi)=\left\{v \in W_{p} ; \partial \varphi(v) \neq \phi\right\} \subset W_{p} \cap V_{1}$ into the set of subsets of $W_{p}^{\prime}$ :

and let

$$
L_{\lambda, \xi}(v)=e_{\lambda, \xi}(v)+B(v)+\partial \varphi(v)
$$

$$
Y_{\xi, n}=\left(\gamma^{-4} n \xi^{3}\right)^{1 / 4} \text { with } \chi=\gamma_{2} \text { from (1.25). }
$$

Then, the following statements hold.

(i) For any $u \in W_{p}^{\prime}$ there exists $v \in \mathscr{D}(\partial \varphi)$ such that

$$
u \in\left(1+\frac{1}{n} L_{\lambda, \xi}\right)(v) \quad(n=1,2, \ldots) .
$$

(ii) Let $v_{i}(i=1,2)$ be solutions of (3.1) with $u=u_{i} \in H$. Then, we have

$$
\left\|\nabla v_{i}\right\| \leq Y_{\xi, n} \text { and }\|\delta v\|^{2}+\frac{\xi}{n}\|\nabla \delta v\|^{2} \leq 2\|\delta u\|^{2},
$$

$$
\text { if } u_{i} \in H_{\lambda, \xi, n}=\left\{u ;\|u\| \leq M_{\lambda, \xi, n}\right\} \text {, where } \delta v=v_{2}-v_{1}, \delta u=u_{2}-u_{1} \text { and }
$$

$$
M_{\lambda, \xi, n}=\left(\frac{2 \xi}{n}\right)^{1 / 2} Y_{\xi, n} \exp \left(\frac{\lambda}{2} Y_{\xi, n}^{c}\right)
$$

Proof. (i) The existence of $v$ follows from Theorem 8.5 of Lions [9, Ch. 2]. In fact, (1.23) implies $c_{1}\|\nabla v\|_{p}^{p} \leq \varphi(v)$ and by definition we have $\left\langle e_{\lambda, \xi}(v), v\right\rangle$ $\geq \xi\|\nabla u\|^{2}$, and hence, it follows that the operator $\left(1+\frac{1}{n} L_{\lambda, \xi}\right)$ is coercive over $W_{p}$ :

$$
\frac{\left.\left\langle v+n^{-1} A(v), v\right\rangle+n^{-1} \varphi(v)\right\rangle}{\|v\|_{W_{p}}} \rightarrow \infty \quad \text { if }\|v\|_{W_{p}} \rightarrow \infty .
$$

(ii) The relation (3.1) yields 


$$
\|v\|^{2}+\frac{2}{n}\left\{\left\langle e_{\lambda, \xi}(v), v\right\rangle+\varphi(v)\right\} \leq\|u\|^{2}
$$

and hence $\left\langle e_{\lambda, \xi}(v), v\right\rangle \leq n\|u\|^{2} / 2$. If $u \in H_{\lambda, \xi, n}$, then

$$
\|\nabla v\|^{2} \exp \left(\lambda\|\nabla v\|^{c}\right) \leq \frac{n}{2 \xi}\|u\|^{2} \leq Y_{\xi, n}^{2} \exp \left(\lambda Y_{\xi, n}^{c}\right) .
$$

So that

$$
\|\nabla v\| \leq Y_{\xi, n}=\left(\gamma^{-4} n \xi^{3}\right)^{1 / 4} .
$$

Keeping in mind the following three inequalities:

$$
\begin{aligned}
\left\langle e_{\lambda, \xi}\left(v_{1}\right)-e_{\lambda, \xi}\left(v_{2}\right), v_{1}-v_{2}\right\rangle & \geq \xi\left\|\nabla\left(v_{1}-v_{2}\right)\right\|^{2}, \\
\left\langle B\left(v_{1}\right)-B\left(v_{2}\right), v_{1}-v_{2}\right\rangle & =-\left\langle B\left(v_{1}-v_{2}\right), v_{1}\right\rangle \\
& \leq r\left\|v_{1}-v_{2}\right\|^{1 / 2}\left\|\nabla\left(v_{1}-v_{2}\right)\right\|^{3 / 2}\left\|\nabla v_{1}\right\|, \\
\left\langle\partial \varphi\left(v_{1}\right)-\partial \varphi\left(v_{2}\right), v_{1}-v_{2}\right\rangle & \geq 0,
\end{aligned}
$$

we can deduce from the relation $u_{i} \in\left(1+\frac{1}{n} L_{\lambda, \xi}\right)\left(v_{i}\right)$ that

$$
\|\delta v\|^{2}+\frac{1}{n}\left\{\xi\|\nabla \delta v\|^{2}-\gamma\|\delta v\|^{1 / 2}\left\|\nabla v_{1}\right\|\|\nabla \delta v\|^{3 / 2}\right\} \leq\langle\delta u, \delta v\rangle .
$$

Applying (1.11) and then (3.4) with $v=v_{1}$, we obtain after a simple calculation

$$
\frac{3}{4}\|\delta v\|^{2}+\frac{\xi}{4 n}\|\nabla \delta v\|^{2} \leq\langle\delta u, \delta v\rangle
$$

from which (3.2) follows by using Schwarz' inequality.

Q. E. D.

There are given $u_{0} \in H$ and $f \in L_{\mathrm{loc}}^{2}(0, \infty ; H)$. Let $a_{n} \in H$ and $f_{n} \in$ $C([0, \infty) ; H)$, and assume that

$$
a_{n} \rightarrow u_{0} \text { in } H \text { and } f_{n} \rightarrow f \text { in } L_{\text {loc }}^{2}(0, \infty ; H) .
$$

We then choose $\lambda$ so that $M_{\lambda, \xi, n}=A_{n} \exp (2 n T)$, that is,

$$
\lambda=2\left(\gamma^{-4} n \xi^{3}\right)^{-c / 4}\left\{2 n T+\log \left(2^{-1 / 2} \gamma n^{1 / 4} \xi^{-5 / 4} A_{n}\right)\right\},
$$

where

$$
A_{n}=\frac{1}{2 n}\left\{\max _{0 \leq t \leq T}\left\|f_{n}(t)\right\|+2 n\left\|a_{n}\right\|\right\} .
$$

It is evident that $\left\|a_{n}\right\| \leq M_{\lambda, \xi, n}$. Substitution of $\xi=\xi_{n}=n^{-\alpha}$ and $T=T_{n}=n^{\beta}$ 
into (3.8) yields $\lambda_{n}$. If we set $M_{n}=M_{\lambda_{n}, \xi_{n}, n}$ and $Y_{n}=Y_{\xi_{n}, n}$, and choose $\alpha$ and $\beta$ as

$$
0<\alpha<\frac{1}{3}\left(1-\frac{4}{c}\right) \text { and } 0<\beta<\frac{c}{4}(1-3 \alpha) \text {, }
$$

it then easily follows that

$$
\begin{aligned}
& \xi_{n} \rightarrow 0, T_{n} \rightarrow \infty \text { and } \lambda_{n} \rightarrow 0 \text { as } n \rightarrow \infty \\
& Y_{n}=\left(\gamma^{-4} n \xi_{n}^{3}\right)^{1 / 4}, \quad M_{n}=A_{n} \exp \left(2 n T_{n}\right) .
\end{aligned}
$$

Proposition 3.2. Let $\varphi_{t} \in \Phi_{p}, p>6 / 5, u_{0} \in H$ and $f \in L_{\mathrm{loc}}^{2}(0, \infty ; H)$, and assume that $a_{n} \in H$ and $f_{n} \in C([0, \infty) ; H)$ satisfy (3.7). Then, there exist sequences $\xi_{n}>0, T_{n}>0, \lambda_{n}>0, Y_{n}>0$, and $M_{n}>0$, satisfying (3.9), such that the following statements hold:

(i ) For any $u$ belonging to

$$
H_{n}=\left\{u \in H ;\|u\| \leq M_{n}\right\}
$$

there corresponds exactly one $v \in \mathscr{D}\left(\partial \varphi_{t}\right)$ such that $u \in\left(1+\frac{1}{n} L_{n}(t, \cdot)\right)(v)$ and $\|\nabla v\| \leq Y_{n}$, where

$$
L_{n}(t, v)=e_{n}(v)+B(v)+\partial \varphi(t, v) \quad \text { with } e_{n}=e_{\lambda_{n}, \xi_{n}} .
$$

(ii) Let $\mathscr{L}_{n}(t, \cdot)$ be Yosida's approximation of $L_{n}$ :

$$
\mathscr{L}_{n}(t, \cdot)=n\left\{1-\left(1+\frac{1}{n} L_{n}(t, \cdot)\right)^{-1}\right\}: H_{n} \rightarrow H .
$$

Then, there exists exactly one function $u_{n}(t)$ in $C^{1}\left(\left[0, T_{n}\right] ; H_{n}\right)$ satisfying

$$
\begin{aligned}
& u_{n}^{\prime}+\mathscr{L}_{n}\left(t, u_{n}(t)\right)=f_{n}(t) \quad \text { in }\left(0, T_{n}\right), \\
& u_{n}(0)=a_{n} .
\end{aligned}
$$

Proof. Choose $\xi_{n}, T_{n}, \lambda_{n}, Y_{n}$ and $M_{n}$ as above. The proof of (i) is an immediate consequence of Proposition 3.1. So we devote our attention to part (ii). Setting $v=\left(1+\frac{1}{n} L_{n}(t, \cdot)\right)^{-1}(u) \in \mathscr{D}\left(\partial \varphi_{t}\right)$, we immediately obtain

$$
\begin{aligned}
& n(u-v)=\mathscr{L}_{n}(t, u) \in L_{n}(t, v) \\
& \|v\|^{2}+\frac{2}{n}\left\{\left\langle e_{n}(v), v\right\rangle+\varphi(t, v)\right\} \leq\|u\|^{2} .
\end{aligned}
$$

Let $b_{n}-M_{n}-\left\|a_{n}\right\|$. We set $U_{n}=\left\{u \in H ;\left\|u-a_{n}\right\| \leq b_{n}\right\}$, which is a subset of $H_{n}$, and define 


$$
\mathscr{F}_{n}(t, u)=f_{n}(t)-\mathscr{L}_{n}(t, u) \text { for }(t, u) \in\left[0, T_{n}\right] \times U_{n} .
$$

We are going to prove that $\mathscr{F}_{n}$ is a continuous function of $\left[0, T_{n}\right] \times U_{n}$ into $H$. With each $t_{i} \in\left[0, T_{n}\right]$ and $u_{i} \in U_{n}(i=1,2)$ we associate $v_{i} \in W_{p} \cap V_{1}$ in a manner that $u_{i} \in v_{i}+\frac{1}{n} L_{n}\left(t_{i}, v_{i}\right)$. Then, we have $\left\|\nabla v_{i}\right\| \leq Y_{n}$ and (3.12) with $u=u_{i}$ and $v=v_{i}$. Therefore, we have

$$
\left\|\mathscr{F}_{n}\left(t_{2}, u_{2}\right)-\mathscr{F}_{n}\left(t_{1}, u_{1}\right)\right\| \leq\left\|f_{n}\left(t_{2}\right)-f_{n}\left(t_{1}\right)\right\|+n(\|\delta u\|+\|\delta v\|),
$$

where $\delta v=v_{2}-v_{1}$ and $\delta u=u_{2}-u_{1}$.

From (3.10) and (3.12) it follows that

$$
\left\langle n\left(u_{i}-v_{i}\right)-e_{n}\left(v_{i}\right)-B\left(v_{i}\right), v_{j}-v_{i}\right\rangle \leq \varphi\left(t_{i}, v_{j}\right)-\varphi\left(t_{i}, v_{i}\right)
$$

for $(i, j)=(1,2)$ and $=(2,1)$. Adding these, we obtain

$$
\begin{aligned}
& \left\langle n \delta(v-u)+\delta e_{n}(v)+\delta B(v), \delta v\right\rangle \\
& \leq \varphi\left(t_{2}, v_{1}\right)-\varphi\left(t_{1}, v_{1}\right)-\varphi\left(t_{2}, v_{2}\right)+\varphi\left(t_{1}, v_{2}\right)
\end{aligned}
$$

and hence, writing the RHS of the above inequality as $\Phi\left(t_{1}, t_{2}\right)$,

$$
n\|\delta v\|^{2}+\xi_{n}\|\nabla \delta v\|^{2}+\left\langle\delta v \cdot \nabla v_{1}, \delta v\right\rangle \leq n\langle\delta u, \delta v\rangle+\Phi\left(t_{1}, t_{2}\right) .
$$

Employing Hölder's inequality and the inequality $\left\|\nabla v_{1}\right\| \leq Y_{n}$ in the term $\left\langle\delta v \cdot \nabla v_{1}, \delta v\right\rangle$, we get analogously as in (3.6)

$$
3\|\delta v\|^{2}+\frac{\xi_{n}}{n}\|\nabla \delta v\|^{2} \leq 4\langle\delta u, \delta v\rangle+4 \Phi\left(t_{1}, t_{2}\right)
$$

So that $\|\delta v\|^{2} \leq 2\|\delta u\|^{2}+4 \Phi$. Hence, combining this with (3.13) concludes the continuity of $\mathscr{F}_{n}$. In fact, (A.2) and (A.3) implies $\Phi\left(t_{1}, t_{2}\right) \rightarrow 0$ as $t_{2} \rightarrow t_{1}$, since $\varphi\left(t_{i}, v_{i}\right) \leq\left\|u_{i}\right\|^{2} \leq\left(b_{n}+\left\|a_{n}\right\|\right)^{2}$.

It is not difficult to see that

$$
\begin{aligned}
& \left\|\mathscr{F}_{n}(t, u)\right\| \leq \alpha_{n}+\beta_{n}\left\|u-a_{n}\right\| \quad \text { with } a_{n}=2 n A_{n} \text { and } \beta_{n}=2 n, \\
& \left\|\mathscr{F}_{n}\left(t, u_{1}\right)-\mathscr{F}_{n}\left(t, u_{2}\right)\right\| \leq 3 n\left\|u_{1}-u_{2}\right\|, u_{i} \in U_{n} \quad(i=1,2)
\end{aligned}
$$

These permit us to apply the method of successive approximation to obtain one and only one $u_{n} \in C^{1}\left(\left[0, T_{n}\right] ; H_{n}\right)$ satisfying (3.11), because $M_{n}=$ $A_{n} \exp \left(2 n T_{n}\right)$ implies

$$
\alpha_{n} \beta_{n}^{-1}\left\{\exp \left(\beta_{n} T_{n}\right)-1\right\} \leq b_{n} .
$$

This completes the proof of part (ii).

Q. E. D. 
Remembering that $u_{n}(t) \in H_{n}$, we define $v_{n}(t) \in \mathscr{D}\left(\partial \varphi_{t}\right)$ by

$$
v_{n}(t)=\left(1+\frac{1}{n} L_{n}(t, \cdot)\right)^{-1}\left(u_{n}(t)\right) .
$$

It then follows from $(3.15)$ that $v_{n} \in C([0, \infty) ; V)$. Furthermore, we have

LEMma 3.1. For each $n$ it follows that

$$
\begin{aligned}
& n\left(u_{n}(t)-v_{n}(t)\right)=\mathscr{L}_{n}\left(t, u_{n}(t)\right) \in L_{n}\left(t, v_{n}(t)\right), \quad 0 \leq t \leq T_{n} \\
& \left\|v_{n}(t)\right\|^{2}+\frac{2}{n}\left\{\left\langle e_{n}\left(v_{n}(t)\right), v_{n}(t)\right\rangle+\varphi\left(t, v_{n}(t)\right)\right\} \leq\left\|u_{n}(t)\right\|^{2}, 0 \leq t \leq T_{n} \\
& \frac{1}{2}\left\|u_{n}(t)\right\|^{2}+\int_{s}^{t}\left\{\left\langle e_{n}\left(v_{n}\right), v_{n}\right\rangle+\varphi\left(\tau, v_{n}\right)\right\} d \tau+\frac{1}{n} \int_{s}^{t}\left\|\mathscr{L}_{n}\left(\tau, u_{n}\right)\right\|^{2} d \tau \\
& \quad \leq \frac{1}{2}\left\|u_{n}(s)\right\|^{2}+\int_{s}^{t}\left\langle f_{n}, u_{n}\right\rangle d \tau, \quad 0 \leq s<t \leq T_{n}
\end{aligned}
$$

and

$$
\begin{aligned}
\text { (P.4) }\left\|u_{n}(t)\right\|^{2}+\int_{0}^{T}\left\{\left\langle e_{n}\left(v_{n}\right), \mathrm{v}_{n}\right\rangle+\varphi\left(t, v_{n}\right)\right\} d t \\
\quad+\frac{1}{n} \int_{0}^{T}\left\|\mathscr{L}_{n}\left(t, u_{n}\right)\right\|^{2} d t \leq K_{T}^{2}
\end{aligned}
$$

for $t, 0 \leq t<T \leq T_{n}$, where $K_{T}$ is a positive constant independent of $t$.

Proof. Properties (P.1) and (P.2) easily follow from (3.12). Keeping in mind

$$
w_{n}(t)=\mathscr{L}_{n}\left(t, u_{n}\right)-B\left(v_{n}\right)-e_{n}\left(v_{n}\right) \in \partial \varphi\left(t, v_{n}\right), \quad u_{n}(0)=a_{n},
$$

we can derive

$$
\begin{aligned}
& \varphi\left(t, v_{n}(t)\right)-\varphi\left(s, v_{n}(s)\right) \\
& \quad \leq\left\langle w_{n}(t), v_{n}(t)-v_{n}(s)\right\rangle+\varphi\left(t, v_{n}(s)\right)-\varphi\left(s, v_{n}(s)\right) .
\end{aligned}
$$

Therefore, (A.3) implies the continuity of $\varphi\left(t, v_{n}(t)\right)$ in $t \geq 0$, because $v_{n} \in$ $C([0, \infty) ; V)$ and $\varphi\left(0, v_{n}(t)\right)$ is bounded in $0 \leq t \leq T_{n}$. On the other hand, from (3.11) and (P.1) it immediately follows that for all $t \geq 0$

$$
\left\langle u_{n}^{\prime}, u_{n}\right\rangle+\left\langle\mathscr{L}_{n}\left(t, u_{n}\right), v_{n}\right\rangle+\frac{1}{n}\left\|\mathscr{L}_{n}\left(t, u_{n}\right)\right\|^{2}=\left\langle f_{n}, u_{n}\right\rangle
$$

Hence, we have by virtue of (3.17) 


$$
\left\langle u_{n}^{\prime}, u_{n}\right\rangle+\left\langle e_{n}\left(v_{n}\right), v_{n}\right\rangle+\varphi\left(t, v_{n}\right)+\frac{1}{n}\left\|\mathscr{L}_{n}\left(t, u_{n}\right)\right\|^{2} \leq\left\langle f_{n}, u_{n}\right\rangle .
$$

Integration over $\Omega \times(s, t)$ of the above gives (P.3). Application of Gronwall's lemma to (P.3) yields (P.4).

Q. E. D.

\section{\$4. Proof of Theorem 1}

For $p>6 / 5$ we define $q=q(p)$ by (1.27). Recalling the fact that $V_{q} \cap V_{1} \subset$ $W_{p}$ (see Lemma 1.1 (ii)), we deduce from (3.11) and (3.17)

$$
\begin{aligned}
& \text { (4.1) } \int_{0}^{T}\left\langle u_{n}^{\prime}, v-v_{n}\right\rangle d t+\int_{0}^{T}\left\langle e_{n}\left(v_{n}\right), v-v_{n}\right\rangle d t+\int_{0}^{T}\left\langle B\left(v_{n}\right), v\right\rangle d t \\
& +\int_{0}^{T}\left\{\varphi(t, v)-\varphi\left(t, v_{n}\right)\right\} d t \geq \int_{0}^{T}\left\langle f_{n}, v-v_{n}\right\rangle d t, \quad v \in C^{1}\left([0, T] ; V_{q} \cap V_{1}\right)
\end{aligned}
$$

for all $n$ such that $T_{n} \geq T$. The proof of Theorem 1 will be accomplished by passage to limit $n \rightarrow \infty$ in (4.1) after a suitable choice of a subsequence of $\left\{u_{n}\right\}$. To do so, using Lemma 3.1, we are going to examine the convergence properties (C.1) (C.7) of the sequences $\left\{u_{n}\right\}$ and $\left\{v_{n}\right\}$.

Lemma 4.1. Suppose $p>6 / 5$. Then, for any $T>0$ we have

$$
\begin{aligned}
& \lim _{n \rightarrow \infty} \int_{0}^{T}\left\|u_{n}-v_{n}\right\|^{2} d t=0, \\
& \lim _{n \rightarrow \infty} \int_{0}^{T}\left\langle e_{n}\left(v_{n}\right), v\right\rangle d t=0, \quad v \in C\left([0, T] ; V_{q} \cap V_{1}\right) .
\end{aligned}
$$

Moreover there exists a subsequence, still denoted by $\{n\}$, of $\{n\}$ such that

$$
\begin{aligned}
& u_{n} \rightarrow u \text { weakly }^{*} \text { in } L^{\infty}(0, T ; H) \\
& v_{n} \rightarrow u \text { weakly }{ }^{*} \text { in } L^{\infty}(0, T ; H) \text { as } n \rightarrow \infty \\
& v_{n} \rightarrow u \text { weakly in } L^{p}\left(0, T ; V_{p}\right)
\end{aligned}
$$

and

$$
\liminf _{n \rightarrow \infty} \int_{0}^{T} \varphi\left(t, v_{n}\right) d t \geq \int_{0}^{T} \varphi(t, u) d t
$$

Proof. Property (C.1) immediately follows from (P.1), (P.2) and (P.4). The boundedness of $\left\{u_{n}\right\}$ and $\left\{v_{n}\right\}$ in Banach spaces $L^{\infty}(0, T ; H)$ and $L^{p}\left(0, T ; V_{p}\right) \cap$ $L^{\infty}(0, T ; H)$, respectively, yields (C.3). Keeping in mind (P.4), we can compute as 
follows:

$$
\begin{aligned}
& \int_{0}^{T}\left\langle e_{n}\left(v_{n}\right), v\right\rangle d t \leq C \int_{0}^{T} \xi_{n}\left\|\nabla v_{n}\right\| \exp \left(\lambda_{n}\left\|\nabla v_{n}\right\|^{c}\right) d t \\
& \quad \leq C \xi_{n}\left\{\int_{E_{n, N}} N^{-1}\left\|\nabla v_{n}\right\|^{2} \exp \left(\lambda_{n}\left\|\nabla v_{n}\right\|^{c}\right) d t+\int_{(0, T) \backslash E_{n, N}} N \exp \left(\lambda_{n} N^{c}\right) d t\right\} \\
& \quad \leq C\left\{K_{T}^{2} / N+\xi_{n} N T \exp \left(\lambda_{n} N^{c}\right)\right\},
\end{aligned}
$$

which leads to (C.2), where

$$
E_{n, N}=\left\{t \in(0, T) ;\left\|\nabla v_{n}(t)\right\|>N\right\} \quad \text { and } \quad C=\sup _{t \in(0, T)}\|\nabla v(t)\| .
$$

The property (C.4) immediately follows from lower-semicontinuity of the mapping $v \rightarrow \int_{0}^{T} \varphi(t, v) d t$

Q. E. D.

Relying on the technique developed by Masuda [10] we can prove

Lemma 4.2. Suppose $p>6 / 5$. Then, there exists a subsequence $\left\{n^{\prime}\right\}$ of $\{n\}$ such that

(C.5) $\lim _{n^{\prime} \rightarrow \infty}\left\langle u_{n^{\prime}}(t), \phi\right\rangle=\langle u(t), \phi\rangle$ uniformly in $[0, T]$ for all $\phi \in H$,

(C.6) $\lim _{n^{\prime} \rightarrow \infty} \int_{0}^{T}\left\|v_{n^{\prime}}-u\right\|_{\Omega_{R}}^{r} d t=0$ for any positive numbers $r$ and $R$,

and

(C.7) $\lim _{n^{\prime} \rightarrow \infty} \int_{0}^{T}\left\langle B\left(v_{n^{\prime}}\right)-B(u), v\right\rangle d t=0$ for all $v \in C\left([0, T] ; V_{q}\right)$,

where $q=q(p), u$ is the same as in (C.3) and $\Omega_{R}=\Omega \cap B_{R}$.

Proof of (C.5). For $\phi \in \mathscr{V}(\Omega)$ let us set $x_{n}(t)=\left\langle u_{n}(t), \phi\right\rangle$. It is easy to see that $\left|x_{n}(t)\right| \leq K_{T}\|\phi\|$ and

$$
\left|x_{n}(t)-x_{n}(s)\right| \leq C_{p}\left\{|t-s|^{\theta}+\int_{s}^{t}\left|\left\langle e_{n}\left(v_{n}\right), \phi\right\rangle\right| d \tau\right\}
$$

for all $0 \leq s<t \leq T_{n}$, where $0<\theta \leq 1$ and $C_{p}$ is a positive constant. This, together with (C.3), allows us to apply the Ascoli-Arzelà theorem, which implies (C.5). 
Proof of (C.6). For the proof we have only to substitute $U=$ "the restriction of $v_{n}-u$ onto $\Omega_{R}$ " into the Friedrichs type inequality: For any $\varepsilon>0$ there exists a positive integer $N$ such that

$$
\|U\|_{\Omega_{R}} \leq \varepsilon\|\nabla U\|_{p, \Omega_{R}}+N \sum_{k=1}^{N}\left|\left\langle\phi_{k}, U\right\rangle_{\Omega_{R}}\right|, \quad U \in W_{\sigma}^{1, p}\left(\Omega_{R}\right),
$$

where $\left\{\phi_{k}\right\}$ is total in $L_{\sigma}^{2}\left(\Omega_{R}\right)$. The proof of (4.2) will be achieved, based on the fact that the injection mapping $W^{1, p}\left(\Omega_{R}\right) \rightarrow L^{2}\left(\Omega_{R}\right)$ is compact if $p>6 / 5$.

Proof of (C.7). From the definition of $B$ we have

$$
\int_{0}^{T}\left\langle B\left(v_{n^{\prime}}\right)-B(u), v\right\rangle d t=-\int_{0}^{T}\left\langle\left(v_{n}-u\right) \otimes v_{n}+u \otimes\left(v_{n}-u\right), \nabla v\right\rangle d t
$$

which is denoted by $I_{n}(\nabla v)$. Here, $u \otimes v$ is a tensor field such that $(u \otimes v)_{i j}=$ $u^{i} v^{j}$. We decompose $I_{n}(\nabla v)$ in the form

where

$$
I_{n}(\nabla v)=I_{n}\left(w_{\lambda}\right)+I_{n}\left(w_{\lambda, \mu}\right)+I_{n}\left(z_{\lambda, \mu}\right),
$$

$$
w_{\lambda}=(1-\eta(\lambda x)) \nabla v, \quad w_{\lambda, \mu}=\eta(\lambda x)\{1-\xi(\mu|\nabla v|)\} \nabla v
$$

and

$$
z_{\lambda, \mu}=\eta(\lambda x) \xi(\mu|\nabla v|) \nabla v
$$

for small $\lambda, \mu>0$. Here $\xi$ and $\eta$ are cut-off function defined by (1.3).

Using Lemma 1.5 and the Dini theorem concerning a monotone decreasing sequence of continuous functions, we can prove that for any $\varepsilon>0$ there exist $\lambda$ and $\mu$ so small that $\left|I_{n}\left(w_{\lambda}\right)\right|<\varepsilon$ and $\left|I_{n}\left(w_{\lambda, \mu}\right)\right|<\varepsilon$. We fix such $\lambda$, $\mu$. Since supp $z_{\lambda, \mu} \subset B_{2 / \lambda}$ and $\left|z_{\lambda, \mu}\right| \leq 2 / \mu$, it follows that

$$
\left|I_{n}\left(z_{\lambda, \mu}\right)\right| \leq \frac{2}{\mu} \int_{0}^{T}\left\|v_{n}-u\right\|_{\Omega_{2 / \lambda}}\left(\left\|v_{n}\right\|+\|u\|\right) d t .
$$

Hence, (C.6) implies

$$
\lim _{n^{\prime} \rightarrow \infty} I_{n^{\prime}}\left(z_{\lambda, \mu}\right)=0 \quad \text { and } \quad \limsup _{n^{\prime} \rightarrow \infty}\left|I_{n^{\prime}}(\nabla v)\right| \leq 2 \varepsilon .
$$

This asserts (C.7).

Q. E. D.

We are now ready to prove Theorem 1. Substituting $n=n^{\prime}$ into (4.1) and letting $n^{\prime} \rightarrow \infty$, we can conclude (2.5) for $v \in C^{1}\left([0, T] ; V_{q} \cap V_{1}\right)$ with the aid 
of $($ C.1 $) \sim($ C.7). In fact, the first term of the LHS of (4.1) is calculated as follows:

$$
\begin{aligned}
\int_{0}^{T}\left\langle u_{n}^{\prime}, v-v_{n}\right\rangle d t & =\int_{0}^{T}\left\{\left\langle v^{\prime}, v-u_{n}\right\rangle+\left\langle u_{n}^{\prime}-v^{\prime}, v-u_{n}\right\rangle\right. \\
& \left.+\left\langle u_{n}^{\prime}, u_{n}-v_{n}\right\rangle\right\} d t \\
\leq \int_{0}^{T}\left\langle v^{\prime}, v-u_{n}\right\rangle d t & -\frac{1}{2}\left(\left\|u_{n}(T)-v(T)\right\|^{2}-\left\|a_{n}-v(0)\right\|^{2}\right) \\
& +\int_{0}^{T}\left\langle f_{n}, \frac{1}{n} \mathscr{L}_{n} u_{n}\right\rangle d t
\end{aligned}
$$

and hence we have by (3.7)

$$
\begin{aligned}
\underset{n^{\prime} \rightarrow \infty}{\limsup } \int_{0}^{T}\left\langle u_{n^{\prime}}^{\prime}, v-v_{n^{\prime}}\right\rangle d t \\
\quad \leq \int_{0}^{T}\left\langle v^{\prime}, v-u\right\rangle d t-\frac{1}{2}\left(\|u(T)-v(T)\|^{2}-\left\|u_{0}-v(0)\right\|^{2}\right) .
\end{aligned}
$$

The other terms of (4.1) will be handled without any difficulty by keeping in mind (C.2), (C.7) and (C.4).

To prove (2.5) for any $v$ belonging to the space $W_{0, T}^{p}$ from (2.6) we extend $v(t)$ outside the interval $[0, T]$ as follows: $v(t)=v(-t)$ for $t<0$ and $=v(2 T-t)$ for $t>T$. Let $v_{\varepsilon}(t)$ be a mollifier defined by (2.14). It is easily seen that $v_{\varepsilon} \in C^{1}\left([0, T] ; V_{q} \cap V_{1}\right), v_{\varepsilon} \rightarrow v \quad$ in $\mathscr{B}_{0, T}^{p} \cap L^{p}\left(0, T ; V_{q}\right)$ and $v_{\varepsilon}^{\prime} \rightarrow v^{\prime}$ weakly ${ }^{*}$ in $\left(\mathscr{B}_{0, T}^{p}\right)^{\prime}$. Substituting $v=v_{\varepsilon}$ into (2.5) and tending $\varepsilon \rightarrow 0$, we have (2.5) for any $v \in W_{0, T}^{p}$ because Lemma 1.4 implies $v \in C([0, \infty) ; H)$ and hence $v_{\varepsilon}(t) \rightarrow v(t)$ uniformly in $C([0, T] ; H)$.

Our next purpose is to prove (2.3). Taking account of (3.17), we can infer from (1.23), using (P.2) and (P.4),

$$
\left|\int_{0}^{T}\left\langle w_{n}, v\right\rangle d t\right| \leq C\left\{\left(\int_{0}^{T}\|\nabla v\|_{p}^{p} d t\right)^{1 / p}+\int_{0}^{T}\|D(v)\|_{1} d t\right\}
$$

for all $v \in \mathscr{B}_{0, T}^{p}$. This guarantees the existence of $\beta$ such that $w_{n} \rightarrow \beta$ weakly ${ }^{*}$ in $\left(\mathscr{B}_{0, T}^{p}\right)^{\prime}$. Thus, it easily follows from (C.7) that

$$
-\int_{0}^{T}\left\langle u, \phi^{\prime}\right\rangle d t=\int_{0}^{T}\langle f-B(u)-\beta, \phi\rangle d t
$$

for all $\phi \in C_{0}^{\infty}\left(0, T ; V_{q} \cap V_{1}\right)$. According to (1.18) and Lemma 1.3 , we can conclude (2.3), observing Lemma 1.6.

The energy inequality (2.7) is an immediate consequence of (P.3) $(s=0)$ and 
(C.2). The inclusion (2.8) easily follows from Lemmas 1.1 and 1.2.

\section{§5. Proof of Theorem 2}

Suppose that $\Omega$ is a domain whose complement is compact. We may therefore assume that there exists a positive constant $R_{0}$ such that $E_{R}=\mathbf{R}^{3} \backslash B_{R}$ is contained in $\Omega$ for all $R>R_{0}$. For a measurable set $M$ we set

$$
\|u\|_{r, M}=\left(\int_{M}|u|^{r} d x\right)^{1 / r} \text { and }\|u\|_{2, M}=\|u\|_{M} .
$$

Let $\varphi(u)=\mu\|D(u)\|_{p}^{p}+g\|D(u)\|_{1}$ with $p \geq 9 / 5$. We assume that $u_{n} \in H$ is the vector field constructed in Proposition 3.2, where $a_{n}=u_{0}$ and $\varphi \in \Phi_{p}$, $p \geq 9 / 5$, for all $n$, and that $v_{n}(t) \in \mathscr{D}\left(\partial \varphi_{t}\right)$ is defined by (3.16). The main purpose of this section is to prove

Proposition 5.1. Suppose that $p \geq 9 / 5$ and $T>0$. For any $\varepsilon>0$ there exists $R>R_{0}$ such that

$$
\limsup _{n \rightarrow \infty} \int_{0}^{T}\left\|u_{n}(t)\right\|_{E_{R}}^{2} d t \leq \varepsilon
$$

Temporarily, let us assume (5.1) to hold. Since

$$
\int_{0}^{T}\left\|u_{n^{\prime}}-u\right\|^{2} d t \leq 2 \int_{0}^{T}\left(\left\|u_{n^{\prime}}-u\right\|_{B_{R}}^{2}+\left\|u_{n^{\prime}}\right\|_{E_{R}}^{2}+\|u\|_{E_{R}}^{2}\right) d t,
$$

it follows from (5.1), (C.1) and (C.5) that

$$
\limsup _{n^{\prime} \rightarrow \infty} \operatorname{LHS} \text { of }(5.2) \leq 4 \varepsilon
$$

which implies by using (P.4)

$$
\int_{0}^{T}\left\|u_{n^{\prime}}-u\right\|^{r} d t \rightarrow 0 \quad \text { as } n^{\prime} \rightarrow \infty
$$

for any $r>0$. Therefore, we can extract a subsequence $\left\{n^{\prime \prime}\right\}$ of $\left\{n^{\prime}\right\}$ so that $u_{n^{\prime \prime}}(s) \rightarrow u(s)$ in $H$ for a.e. $s>0$. Substituting $n=n^{\prime \prime}$ into (P.3) and letting $n^{\prime \prime} \rightarrow \infty$, we obtain (2.22).

Before proving the proposition we prepare a few lemmas. For $0<\lambda<1$ such that $1 / \lambda>R_{0}$ we introduce a cut-off function:

$$
\zeta_{\lambda}(x)=\{1-\eta(\lambda x)\}^{2 p}(\text { see }(1.3) \text { for } \eta(x))
$$


and the fundamental solution of $\lambda-\Delta$ :

$$
F_{\lambda}=\frac{1}{4 \pi|x|} \exp (-\sqrt{\lambda}|x|)
$$

Like (1.6) we define a mapping $v \rightarrow v_{\lambda}$ :

$$
v_{\lambda}=\operatorname{rot}\left\{\zeta_{\lambda}\left(F_{\lambda} *\left(\zeta_{\lambda} \operatorname{rot} v\right)\right)\right\}, \quad 1 / \lambda>R_{0} .
$$

After a simple calculation we obtain

$$
v_{\lambda}=\zeta_{\lambda}\left\{\left(\delta-\lambda F_{\lambda}\right) *\left(\zeta_{\lambda} v\right)\right\}+R_{\lambda} v
$$

where

$$
\begin{array}{r}
R_{\lambda} v=\zeta_{\lambda}\left\{F_{\lambda} * \operatorname{rot}\left(v \times \nabla \zeta_{\lambda}\right)\right\}+\nabla \zeta_{\lambda} \times\left\{F_{\lambda} * \operatorname{rot}\left(\zeta_{\lambda} v\right)\right\} \\
+\nabla \zeta_{\lambda} \times\left\{F_{\lambda} *\left(v \times \nabla \zeta_{\lambda}\right)\right\}
\end{array}
$$

Using the inequality (1.4), the identity (1.8) and the estimations with respect to $F$ :

we easily see that if $v$ is in $H$ (or $V_{r}, r \geq 1$ ), then so is $v_{\lambda}$, where and in what follows $C$ denotes various positive constants not depending on $\lambda$. More precisely we can show quite easily

Lemma 5.1. For any $v \in C_{0}^{\infty}\left(\mathbf{R}^{3}\right)^{3}$ we have

$$
\begin{aligned}
& \left\|R_{\lambda} v\right\| \leq C \lambda^{1 / 2}\|v\|, \quad\left\|\nabla R_{\lambda} v\right\| \leq C \lambda\|v\|, \\
& \left\|\nabla R_{\lambda} v\right\|_{r} \leq C \lambda^{1 / 2}\left(\|\nabla v\|_{r}+\|v\|\right), \quad r>6 / 5, \\
& \left\|D\left(R_{\lambda} v\right)\right\|_{1} \leq C \lambda^{1 / 2}\|D(v)\|_{1} .
\end{aligned}
$$

Proof. The proof of (5.7) is evident. Without any difficulty we can show that

$$
\left\|D\left(R_{\lambda} v\right)\right\|_{r} \leq C_{r} \lambda^{1 / 2}\left(\|D(v)\|_{r}+\lambda\|v\|_{r, B_{2 / \lambda}}\right)
$$

for all $r \geq 1$. Consequently, the use of (1.1) and Lemma 1.2 imply (5.8). By Hölder's inequality we have

$$
\|v\|_{1, B_{2 / \lambda}} \leq C \lambda^{-1}\|v\|_{3 / 2} .
$$

Hence, the proof of (5.9) is achieved with the aid of (1.2).

Q. E. D. 
Lemma 5.2. Suppose that $p \geq 9 / 5$. Then, we have

$$
\left|\left\langle B(v), v_{\lambda}\right\rangle\right| \leq C \lambda^{1 / 2}\|v\|^{a}\|\nabla v\|_{q}^{b}, \quad v \in \mathcal{V},
$$

where $a, b$ and $q$ are positive numbers such that $a+b=3, b \leq q$ and $q=p$ for $p<3$ and $=2$ for $p \geq 3$.

Proof. After a simple calculation we obtain from (5.4) that

$$
\begin{aligned}
\left\langle B(v), v_{\lambda}\right\rangle=\left\langle\xi_{\lambda} v^{i} v^{j},\right. & \left.\lambda \nabla_{i} F_{\lambda} *\left(\zeta_{\lambda} v^{j}\right)\right\rangle \\
& -\left\langle v^{i} v^{j} \nabla_{i} \zeta_{\lambda},\left(\delta-\lambda F_{\lambda}\right) *\left(\zeta_{\lambda} v^{j}\right)\right\rangle-\left\langle v^{i} v^{j}, \nabla_{i}\left(R_{\lambda} v^{j}\right)\right\rangle
\end{aligned}
$$

and hence, using (1.4), (5.6) and (5.7), we get

$$
\left|\left\langle B(v), v_{\lambda}\right\rangle\right| \leq C \lambda^{1 / 2}\|v\|\|v\|_{4}^{2} .
$$

Assume that $9 / 5 \leq p<3$. Then, $2<4<p^{*}$. Using (1.10) and (1.2), we obtain

$$
\|v\|_{4}^{2} \leq C\|v\|^{2-\beta}\|\nabla v\|_{p}^{\beta} \quad \text { with } \beta=3 p /(5 p-6) .
$$

Evidently, $p \geq 9 / 5$ implies $\beta \leq p$. We now suppose $p \geq 3$. Instead of (5.13) the inequality:

$$
\|v\|_{4}^{2} \leq C\|v\|^{1 / 2}\|\nabla v\|^{3 / 2}
$$

is adopted. Combining (5.12) with (5.13)-(5.14), we arrive at (5.11).

Q. E. D.

Let $a \geq 1$ and $q \geq 1$. Set $z_{\lambda}=\zeta_{\lambda}^{1 / p}$. Using Hölder's inequality, we have for $h \in L^{q}$

$$
\begin{gathered}
\left|z_{\lambda}^{a}\left(F_{\lambda} * h\right)-F_{\lambda} *\left(z_{\lambda}^{a} h\right)\right| \leq \frac{1}{4 \pi} \int \frac{1}{|x-y|} e^{-\sqrt{\lambda}|x-y|}\left|z_{\lambda}^{a}(x)-z_{\lambda}^{a}(y)\right||h(y)| d y \\
\leq C \lambda \int e^{-\sqrt{\lambda}|x-y|}|h(y)| d y \leq C \lambda^{1-3 / 2 q^{\prime}}\left(\int e^{-\sqrt{\lambda}|x-y|}|h(y)|^{q} d y\right)^{1 / q} .
\end{gathered}
$$

Hence,

$$
\left\|z_{\lambda}^{a}\left(F_{\lambda} * h\right)-F_{\lambda} *\left(z_{\lambda}^{a} h\right)\right\|_{q} \leq C \lambda^{1-3 / 2 q^{\prime}-1 / 2 q}\|h\|_{q} \leq C \lambda^{-1 / 2}\|h\|_{q} .
$$

With the aid of (5.15) we shall prove the last two lemmas.

Lemma 5.3. Let $\phi_{p}(v)=\|D(v)\|_{p}^{p}, \quad p \geq 9 / 5$. Then,

$$
-\left\langle\partial \phi_{p}(v), v_{\lambda}\right\rangle \leq C \lambda^{1 / 2}\left(\|\nabla v\|_{p}^{p}+\|v\|\|\nabla v\|_{p}^{p-1}\right), \quad v \in \mathscr{D}(\partial \varphi) .
$$


Proof. In view of (5.4) we have

$$
\begin{aligned}
& D\left(v_{\lambda}\right)=\zeta_{\lambda}\left\{\left(\delta-\lambda F_{\lambda}\right) *\left(\zeta_{\lambda} D(v)\right\}\right. \\
& -\left\{\zeta_{\lambda}\left(\Delta F_{\lambda} *\left(\left[D, \zeta_{\lambda}\right] v\right)\right)+\left[D, \zeta_{\lambda}\right]\left(\Delta F_{\lambda} *\left(\zeta_{\lambda} v\right)\right)-D\left(R_{\lambda} v\right)\right\}=X-Y
\end{aligned}
$$

and hence,

$$
\text { the LHS of }(5.16)=-p\left\langle|D(v)|^{p-2} D(v), X-Y\right\rangle \text {, }
$$

where $[D, \zeta] u=D(\zeta u)-\zeta D(u)$ and hence

$$
([D, \zeta] u)_{i j}=\left\{\left(\nabla_{i} \zeta\right) u^{j}+\left(\nabla_{j} \zeta\right) u^{i}\right\} / 2 .
$$

Firstly, we have in view of (5.15)

$$
\begin{gathered}
-p\left\langle|D(v)|^{p-2} D(v), X\right\rangle \\
=-p\left\|z_{\lambda}^{2} D(v)\right\|_{p}^{p}+p\left\langle|D(v)|^{p-2} D(v), z_{\lambda}^{2 p-2}\left\{\lambda F_{\lambda} *\left(z_{\lambda}^{2} D(v)\right)\right\}\right\rangle \\
+p\left\langle|D(v)|^{p-2} D(v), \lambda F_{\lambda} *\left(z_{\lambda}^{2 p} D(v)\right)-z_{\lambda}^{2 p-2}\left\{\lambda F_{\lambda} *\left(z_{\lambda}^{2} D(v)\right\}\right.\right. \\
\left.+z_{\lambda}^{p}\left\{\lambda F_{\lambda} * z_{\lambda}^{a} D(v)\right\}-\lambda F_{\lambda} *\left(z_{\lambda}^{2 p} D(v)\right)\right\rangle \\
\leq C\|D(v)\|_{p}^{p-2} \lambda^{1 / 2}\|D(v)\|_{p} \leq C \lambda^{1 / 2}\|\nabla v\|_{p}^{p} .
\end{gathered}
$$

By the same argument as is employed in the proof of (5.8) we obtain

$$
p\left\langle|D(v)|^{p-2} D(v), Y\right\rangle \leq C \lambda^{1 / 2}\|D(v)\|_{p}^{p-1}\left(\|\nabla v\|_{p}+\|v\|\right),
$$

which concludes (5.16).

Q. E. D.

Lemma 5.4. Let $\phi_{1}(v)=\|D(v)\|_{1}$. Then,

$$
\left|\left\langle\partial \phi_{1}(v), v_{\lambda}\right\rangle\right| \leq C \lambda^{1 / 2}\|D(v)\|_{1}, \quad v \in \mathscr{D}(\partial \varphi) .
$$

Proof. Let $w \in \partial \varphi_{1}(v)$. Then, we have

$$
\left\langle w, v_{\lambda}\right\rangle=\left\langle w, \zeta_{\lambda}\left\{\left(\delta-\lambda F_{\lambda}\right) * \zeta_{\lambda} v\right\}\right\rangle+\left\langle w, R_{\lambda} v\right\rangle=A+B .
$$

Inserting $\phi=v-t \zeta_{\lambda}\left\{\left(\delta-\lambda F_{\lambda}\right) * \zeta_{\lambda} v\right\}(0<t<1)$ into the inequality $\langle w, \phi-v\rangle \leq \varphi_{1}(\phi)-\varphi_{1}(v)$, we have

$$
t A \geq \varphi_{1}(v)-\varphi_{1}(\phi)=\|D(v)\|_{1}-\|D(\phi)\|_{1} .
$$

A similar calculation as in (5.17) leads to

$$
\begin{gathered}
D(\phi)=\left(1-t \zeta_{\lambda}^{2}\right) D(v)+t \lambda F_{\lambda} * \zeta_{\lambda}^{2} D(v) \\
+t\left\{\zeta_{\lambda}\left(\lambda F_{\lambda} * \zeta_{\lambda} D(v)\right)-\lambda F_{\lambda} * \zeta_{\lambda}^{2} D(v)\right\}+t \zeta\left(\zeta_{\lambda}\right)\left(\Delta F_{\lambda} * \zeta_{\lambda} v\right) \\
+t \zeta_{\lambda}\left(\Delta F_{\lambda} * D\left(\zeta_{\lambda}\right) v\right) .
\end{gathered}
$$


Making use of (5.15), we get

$$
\begin{aligned}
\|D(\phi)\|_{1} \leq\|D(v)\|_{1} & +t C \lambda^{1 / 2}\left\|\zeta_{\lambda} D(v)\right\|_{1} \\
& +t\left\|D\left(\zeta_{\lambda}\right)\left\{F_{\lambda} * \Delta\left(\zeta_{\lambda} v\right)\right\}\right\|_{1}+t\left\|\zeta_{\lambda}\left\{F_{\lambda} * \Delta\left(D\left(\zeta_{\lambda}\right) v\right)\right\}\right\|_{1} .
\end{aligned}
$$

Exactly as in (5.9) we have (5.18).

Q. E. D.

Proof of Proposition 5.1. Multiplying (3.17) by $u_{n, \lambda}$ and integrating over $\Omega \times(0, t)$, we obtain, keeping in mind (3.11), that

$$
\begin{aligned}
\int_{0}^{t}\left\langle u_{n}^{\prime}, u_{n, \lambda}\right\rangle d \tau & =\int_{0}^{t}\left\langle f_{n}, u_{n, \lambda}\right\rangle d \tau-\frac{1}{n} \int_{0}^{t}\left\langle\mathscr{L}_{n}\left(u_{n}\right),\left(\mathscr{L}_{n}\left(u_{n}\right)\right)_{\lambda}\right\rangle d \tau \\
& -\int_{0}^{t}\left\langle B\left(v_{n}\right)+e_{n}\left(v_{n}\right)+\partial \psi_{p}\left(v_{n}\right)+w_{n}, v_{n, \lambda}\right\rangle d \tau
\end{aligned}
$$

where $w_{n}(t) \in \partial \varphi_{1}\left(v_{n}(t)\right)$. Since

$$
\left\langle u_{n}^{\prime}, u_{n, \lambda}\right\rangle=\frac{1}{4} \frac{d}{d t}\left\langle\zeta_{\lambda} \operatorname{rot} u_{n}, F_{\lambda} *\left(\zeta_{\lambda} \operatorname{rot} u_{n}\right)\right\rangle
$$

we have

$$
2 \int_{0}^{t}\left\langle u_{n}^{\prime}, u_{n, \lambda}\right\rangle d \tau=\left\langle u_{n}(t), u_{n, \lambda}(t)\right\rangle-\left\langle u_{n}, u_{n, \lambda}\right\rangle
$$

On the other hand we obtain from (5.4), (5.6) and (5.7) that

$$
\begin{aligned}
-\left\langle u_{n}, u_{n, \lambda}\right\rangle & +\left\|\zeta_{\lambda} u_{n}\right\|^{2}=\left\langle u_{n}-v_{n}+v_{n}, \zeta_{\lambda}\left(\lambda F_{\lambda} *\left(\zeta_{\lambda} u_{n}\right)\right)\right\rangle-\left\langle u_{n}, R_{\lambda} u_{n}\right\rangle \\
& \leq\left\|u_{n}-v_{n}\right\|\left\|u_{n}\right\|+C \lambda^{1 / 2}\left\|u_{n}\right\|^{2}+\left\|\zeta_{\lambda} v_{n} * \lambda F_{\lambda}\right\|\left\|u_{n}\right\| .
\end{aligned}
$$

Therefore, we get, using (P.4),

$$
\begin{aligned}
\left\|\zeta_{\lambda} u_{n}\right\|^{2} & \leq 2 \int_{0}^{t}\left\langle u_{n}^{\prime}, u_{n, \lambda}\right\rangle d s+\left\|\zeta_{\lambda} u_{n}\right\|^{2}+C \lambda^{1 / 2}\left\|u_{0}\right\|^{2} \\
& +K_{T}\left(\left\|u_{n}(t)-v_{n}(t)\right\|+\left\|\zeta_{\lambda} v_{n}(t) * \lambda F_{\lambda}\right\|\right)+C K_{T} \lambda^{1 / 2}
\end{aligned}
$$

for all $t \leq T$.

For the proof of the proposition it is sufficient to establish

$$
\underset{n \rightarrow \infty}{\lim \sup } \int_{0}^{T}\left\|\zeta_{\lambda} u_{n}(t)\right\|^{2} d t \rightarrow 0 \quad \text { as } \lambda \rightarrow 0 .
$$

Applying (1.4) with $r=2, p=3 / 2$ and $q=6 / 5$, we obtain, keeping in mind 


$$
\left\|\zeta_{\lambda} v_{n} * \lambda F_{\lambda}\right\| \leq\left\|v_{n}\right\|_{3 / 2}\left\|\lambda F_{\lambda}\right\|_{6 / 5} \leq C \lambda^{1 / 10}\left\|D\left(v_{n}\right)\right\|_{1} \text {. }
$$

Thus, we have only to pay attention to each term of the RHS of (5.19). From (5.7) it immediately follows that

$$
\begin{gathered}
\text { (5.25) } \int_{0}^{t}\left\langle f_{n}, u_{n, \lambda}\right\rangle d s \leq 2 \int_{0}^{T}\left\|\zeta_{\lambda} f_{n}\right\| \zeta_{\lambda} u_{n}\left\|d s+C \lambda^{1 / 2} \int_{0}^{T}\right\| f_{n}\|\| u_{n} \| d s \\
\leq 2 K_{T} \int_{0}^{T}\left(\left\|f_{n}-f\right\|+\left\|\zeta_{\lambda} f\right\|\right) d s+C K_{T} \lambda^{1 / 2} \int_{0}^{T}\left\|f_{n}\right\| d s, \\
(5.26)-\frac{1}{n} \int_{0}^{t}\left\langle\mathscr{L}_{n}\left(u_{n}\right),\left(\mathscr{L}_{n}\left(u_{n}\right)_{\lambda}\right\rangle d s \leq C \lambda^{1 / 2} \frac{1}{n} \int_{0}^{T}\left\|\mathscr{L}_{n}\left(u_{n}\right)\right\|^{2} d s \leq C K_{T}^{2} \lambda^{1 / 2},\right.
\end{gathered}
$$

and

$$
-\int_{0}^{t}\left\langle e\left(v_{n}\right), v_{n, \lambda}\right\rangle d s \leq C \lambda \int_{0}^{T} \xi_{n}\left\|v_{n}\right\|\left\|\nabla v_{n}\right\| \exp \left(\lambda_{n}\left\|\nabla v_{n}\right\|^{c}\right) d s
$$

Here, we used the positively of $\delta-\lambda F_{\lambda}$ :

$$
\left\langle h,\left(\delta-\lambda F_{\lambda}\right) * h\right\rangle \geq 0, \quad h \in L^{2} .
$$

From Lemma 5.2 it follows that

$$
-\int_{0}^{t}\left\langle B\left(v_{n}\right), v_{n, \lambda}\right\rangle d s \leq C \lambda^{1 / 2} \int_{0}^{T}\left\|v_{n}\right\|^{2}\left\|\nabla v_{n}\right\|_{q}^{b} d s \leq C C_{T} \lambda^{1 / 2}
$$

Lemmas 5.3 and 5.4 lead to

$$
\begin{gathered}
-\int_{0}^{t}\left\langle\partial \varphi\left(v_{n}\right)+w_{n}, v_{n, \lambda}\right\rangle d s \\
\leq C \lambda^{1 / 2} \int_{0}^{T}\left(\left\|\nabla v_{n}\right\|_{p}^{p}+\left\|v_{n}\right\|\left\|\nabla v_{n}\right\|_{p}^{p-1}+\left\|D\left(v_{n}\right)\right\|_{1}\right) d s \leq C C_{T} \lambda^{1 / 2} .
\end{gathered}
$$

Thanks to (5.22), we can prove (5.23) by virtue of $(5.24) \sim(5.29)$.

\section{\$6. Proof of Theorem 3}

We first observe that functional $\varphi_{t}(u)=\varphi(t, u)$ defined by (2.23) satisfies (A.1) $\sim$ (A.3) with $p=2$ if $\mu \in \mathcal{M}$ and $g \in \mathscr{G}$. Applying Proposition 3.2 with $a_{n}=u_{0}+\frac{\chi}{n}$ and $f_{n}=f$, we can find sequences $\left\{\lambda_{n}\right\},\left\{T_{n}\right\},\left\{\xi_{n}\right\},\left\{Y_{n}\right\}$ and $\left\{M_{n}\right\}$ satisfying (3.9) and that for any $u \in H_{n}=\left\{u \in H ;\|u\| \leq M_{n}\right\}$ and any $t \geq 0$ 
there exists exactly one $v \in V$ such that $u \in\left(1+\frac{1}{n} L_{n}(t, \cdot)\right)(v)$ and $\|\nabla v\| \leq Y_{n}$, where

$$
\begin{gathered}
L_{n}(t, v)=B(v)+e_{n}(v)+\partial \varphi_{n}(t, v) \\
\varphi_{n}(t, v)=\varphi(t, v)-\varepsilon_{n}\|D(v)\|^{2} \text { with } \varepsilon_{n}=\xi_{n} \exp \left(\lambda_{n}\left\|\nabla u_{0}\right\|^{c}\right) .
\end{gathered}
$$

Moreover, setting

$$
\mathscr{L}_{n}(t, u)=n\left\{1-\left(1+\frac{1}{n} L_{n}(t, \cdot)\right)^{-1}\right\}(u): H_{n} \rightarrow H,
$$

we obtain one and only one function $u_{n} \in C^{1}\left(\left[0, T_{n}\right] ; H_{n}\right)$ satisfying

$$
\begin{aligned}
& u_{n}^{\prime}(t)+\mathscr{L}_{n}\left(t, u_{n}(t)\right)=f(t) \quad \text { in } t \in\left(0, T_{n}\right), \\
& u_{n}(0)=a_{n} .
\end{aligned}
$$

We then define $v_{n}(t)$ as in (3.16):

$$
v_{n}(t)=\left\{1+\frac{1}{n} L_{n}(t, \cdot)\right\}^{-1}\left(u_{n}(t)\right)
$$

From (3.15) it immediately follows that $v_{n} \in C\left(\left[0, T_{n}\right] ; V\right)$ for all $n$. We can further prove that

$$
v_{n}(0)=u_{0} \text { and } \mathscr{L}_{n}\left(0, u_{n}(0)\right)=\chi .
$$

In fact, observing (2.30) and $\partial \varphi\left(t, u_{0}\right)=e_{n}\left(u_{0}\right)+\partial \varphi_{n}\left(t, u_{0}\right)$, we have $\chi \in L_{n}\left(0, u_{0}\right)$ and hence $u_{n}(0)=u_{0}+\frac{1}{n} \chi \in\left(1+\frac{1}{n} L_{n}(0, \cdot)\right)\left(u_{0}\right)$.

Analogously as in Theorem 1 we can find a weak solution $u$ of (2.25)-(2.26). Corollary 1 says that $u$ is a strong solution of (2.25)-(2.26) as well if it satisfies (2.32). So we have only to establish the regularity properties (2.32) and (2.33).

We first consider a solution $u \in V$ of a stationary problem:

$$
\langle B(u), v-u\rangle+\varphi(t, v)-\varphi(t, u) \geq\langle h, v-u\rangle, \quad v \in V
$$

for $t \geq 0$ and $h \in L^{\infty}(\Omega)^{3}$. It is easily seen from the Hahn-Banach theorem and Temam [17, p.14] that there exist $\pi \in L^{2}(\Omega)$, a constant $c=c(\Omega)$ and $m=\left(m_{i j}\right)_{i, j=1}^{3}$ with $m_{i j} \in L^{\infty}(\Omega)$ and $|m| \leq g_{1}$ such that

$$
\begin{gathered}
-\nabla \cdot(2 \mu D(u)+m)+B(u)+\nabla \pi=h, \\
\|\pi\| \leq c\left(\|h\|+\|B(u)\|_{V^{\prime}}+\|\mu \nabla u\|+g_{1}\right) .
\end{gathered}
$$


Moreover, we can establish the regularity of $u$ as in Kim [8], making use of Cattabriga's result concerning the regularity of solutions of the Stokes equation (see [4]).

Lemma 6.1. Let $u \in V$ be a solution of (6.5) and assume that a satisfies (2.27). Then, there exists a positive constant $C_{0}$ depending only on $a$ and $\Omega$ such that

$$
\|\nabla u\|_{a} \leq C_{0} \nu_{0}\left(\|\nu \nabla \mu(t)\|_{\alpha}+1\right)\left(\|h\|+\|u\|_{\alpha}\|\nabla u\|+g_{1}+\mu_{0}\|\nabla u\|\right),
$$

where $\nu=1 / \mu(t)$ and $\nu_{0}=1 / \mu_{0}$.

Proof. We begin by rewriting (6.6) as

$$
-\Delta u+\nabla(\nu \pi)=\nu \nabla \mu \cdot\left(2 D(u)-\nu \pi I_{d}+\nu m\right)+\nabla \cdot(\nu m)+\nu h-\nu B(u),
$$

where $I_{d}$ denotes the identity tensor. The inequality (6.8) is then an easy consequence of (6.7) and the inequality due to [4] (see also [17, p. 35]):

$$
\begin{aligned}
& \|\nabla u\|_{a}+\|\nu \pi\|_{a} \leq C\|\nu \nabla \mu\|_{\alpha}(\|\nabla u\|+\|\nu \pi\|+\|\nu m\|) \\
& \quad+C\left(\|\nu m\|_{a}+\|\nu h\|+\nu_{0}\|u\|_{\alpha}\|\nabla u\|\right) .
\end{aligned}
$$

Q. E. D.

Lemma 6.2. Let $N$ be the largest integer in the set of integers $<b / 2$ and let us define finite sequences $\left\{a_{n}\right\}_{n=0}^{N}$ and $\left\{r_{n}\right\}_{n=0}^{N}$ by

$$
\frac{1}{a_{n}}=\frac{1}{2}-\frac{n}{b} \text { and } \quad \frac{1}{r_{n}}=\frac{1}{a_{n}}+\frac{1}{3} \text { for } n \leq N \text {. }
$$

Let $q>a$, and assume that $a_{n_{0}-1}<q \leq a_{n_{0}}$ (or $a_{N}<q$ ) and $1 / r=1 / q+1 / 3$. Then, for any solution $u$ of (6.5) the following estimates hold.

$$
\|\nabla u\|_{q}+\|\nu \pi\|_{q} \leq c_{l}\left\{P^{l}(\|\nabla u\|+\|\nu \pi\|)+\frac{P^{l}-1}{P-1} Q_{r}\right\}
$$

where $l=n_{0}$ or $N+1, c_{l}$ is a positive constant depending only on $\alpha, l$ and $\Omega$, and

$$
P=\|\nu \nabla \mu(t)\|_{\alpha}+\nu_{0}\|u\|_{\alpha}, \quad Q_{r}=\nu_{0}\left\{g_{1}\left(1+\|\nu \nabla \mu(t)\|_{\alpha}\right)+\|h\|_{r}\right\} .
$$

Proof. Since $1 / \alpha+1 / b=1 / 3$, it follows that $1 / \alpha+1 / a_{n-1}=1 / r_{n}$ for all $n \geq N$. Hence

$$
L^{r_{n}}(\Omega) \subset W^{-1, a_{n}}(\Omega) \text { and }\|\nu B(u)\|_{r_{n}} \leq \nu_{0}\|u\|_{\alpha}\|\nabla u\|_{a_{n-1}} .
$$

Like (6.9), we obtain 


$$
\begin{aligned}
\|\nabla u\|_{a_{n}}+\|\nu \pi\|_{a_{n}} \leq & C_{n}\|\nu \nabla \mu\|_{\alpha}\left(\|\nabla u\|_{a_{n-1}}+\|\nu \pi\|_{a_{n-1}}+\|\nu m\|_{a_{n-1}}\right) \\
& +C_{n}\left(\|\nu m\|_{\alpha_{n}}+\|\nu h\|_{r_{n}}+\nu_{0}\|u\|_{\alpha}\|\nabla u\|_{a_{n-1}}\right)
\end{aligned}
$$

for all $n \leq N$, where $C_{n}$ is a positive constant depending only on $\alpha, n$ and $\Omega$. Therefore, we have

$$
\|\nabla u\|_{a_{n}}+\|\nu \pi\|_{a_{n}} \leq C_{n}^{\prime}\left\{P\left(\|\nabla u\|_{a_{n-1}}+\|\nu \pi\|_{a_{n-1}}\right)+Q_{r_{n}}\right\},
$$

from which it follows by induction on $n$ that

$$
\|\nabla u\|_{a_{n}}+\|\nu \pi\|_{a_{n}} \leq c_{n}\left\{P^{n}(\|\nabla u\|+\|\nu \pi\|)+\frac{P^{n}-1}{P-1} Q_{r_{n}}\right\} .
$$

The proof of (6.11) is readily achieved.

Q. E. D.

We now return to (6.2) and (6.3).

Proposition 6.1. Let $T>0$. Suppose that there exists a positive constant $E$ satisfying one of the following conditions

$$
\text { (i) }\left\{\begin{array} { l } 
{ \gamma _ { 0 } ^ { 5 } / \gamma _ { 0 } ^ { 4 } > c _ { 0 } A _ { T } E } \\
{ \mu _ { 0 } \| \nabla u _ { 0 } \| ^ { 2 } < E }
\end{array} \text { and (ii) } \left\{\begin{array}{l}
\mu_{0}^{3}>T^{1 / 2} E \\
\mu_{0}\left\|\nabla u_{0}\right\|^{2}<E
\end{array}\right.\right.
$$

and define

$$
T_{n}(E)=\sup \left\{T^{*} ; \mu_{0}\left\|\nabla v_{n}(t)\right\|^{2}<E, 0 \leq t<T^{*} \leq T\right\} .
$$

Then, there exists a positive integer $n_{0}$ such that $T_{n}(E)>0$ and

$$
\left\|u_{n}^{\prime}(t)\right\|^{2}+\frac{\mu_{n, 0}}{4} \int_{0}^{t}\left\|\nabla v_{n}^{\prime}\right\|^{2} d t \leq I_{T}+J_{T}\left(\mu_{0} E+\mu_{0}^{\lambda-2} A_{T}^{\lambda} E^{2-\lambda}\right),
$$

for all $t \leq T_{n}(E)$ and all $n \geq n_{0}$, where $\mu_{n, 0}=\mu_{0}-\varepsilon_{n}$, and $A_{T}, I_{T}, J_{T}$ are the same as in Theorem 3.

Proof. From (6.2) and (6.3) it follows that

$$
\begin{aligned}
& \left\langle e_{n}\left(v_{n}(t)\right)+B\left(v_{n}(t)\right), v-v_{n}(t)\right\rangle+2\left\langle\mu_{n}(t) D\left(v_{n}(t)\right), D\left(v-v_{n}(t)\right)\right\rangle \\
& +\int_{\Omega} g(t)\left(|D(v)|-\left|D\left(v_{n}(t)\right)\right|\right) d x \geq\left\langle f(t)-u_{n}^{\prime}(t), v-v_{n}(t)\right\rangle, \quad v \in V,
\end{aligned}
$$

where $\mu_{n}(t)=\mu(t)-\varepsilon_{n}$. Inserting $v=v_{n}(t+h)$, we obtain after a simple calculation 


$$
\begin{gathered}
\left\langle\delta_{h} e_{n}\left(v_{n}\right)+\delta_{h} B\left(v_{n}\right), \delta_{h} v_{n}\right\rangle+2\left\langle\delta_{h}\left(\mu_{n} D\left(v_{n}\right)\right), D\left(\delta_{h} v_{n}\right)\right\rangle \\
\leq\left\langle\delta_{h}\left(f-u_{n}^{\prime}\right), \delta_{h} v_{n}\right\rangle-\left\langle\delta_{h} g, D\left(\delta_{h} v_{n}\right)\right\rangle,
\end{gathered}
$$

where $\delta_{h} u=\{u(t+h)-u(t)\} / h$. Keeping in mind $f-u_{n}^{\prime}=\mathscr{L}_{n}\left(t, u_{n}\right)$ and $\delta_{h} v_{n}=\delta_{h} u_{n}-\frac{1}{n} \delta_{h} \mathscr{L}_{n}\left(t, u_{n}\right)$ and using Schwarz' inequality, we get

$$
\begin{aligned}
& \frac{d}{d t}\left\|\delta_{h} u_{n}\right\|^{2}+\left\|\sqrt{\mu_{n}} D\left(\delta_{h} v_{n}\right)\right\|^{2}-2\left\langle B\left(\delta_{h}\left(v_{n}\right), v_{n}(t)\right\rangle\right. \\
\leq & 2\left\|\sqrt{\nu \mu_{n}} \delta_{h} \mu \cdot D\left(v_{n}\right)\right\|^{2}+2\left\langle\delta_{h} f, \delta_{h} u_{n}\right\rangle+\left\|\sqrt{\nu_{n}} \delta_{h} g\right\|^{2} .
\end{aligned}
$$

We first suppose (i) of (6.12) to hold. Then, (6.16), together with (2.27) and (2.28), leads to

$$
\begin{gathered}
\frac{d}{d t}\left\|\delta_{h} u_{n}\right\|^{2}+\frac{1}{4}\left(2 \mu_{n, 0}-\gamma_{0}\left\|v_{n}\right\|_{3}\right)\left\|\nabla \delta_{h} v_{n}\right\|^{2} \\
\leq\left\|\delta_{h} f_{n}\right\|+2\left\|\nu_{n} \delta_{h} \mu\right\|_{b}^{2}\left\|\sqrt{\mu_{n}} \nabla v_{n}\right\|_{a}^{2}+\left\|\sqrt{\nu_{n}} \delta_{h} g\right\|^{2}+\left\|\delta_{h} f\right\|\left\|\delta_{h} u_{n}\right\|^{2},
\end{gathered}
$$

where $v_{n}=1 / \mu_{n}$.

On the other hand, from (6.15) with $v=0$ it immediately follows that

$$
\frac{1}{2} \frac{d}{d t}\left\|u_{n}\right\|^{2}+\varphi_{n}\left(t, v_{n}\right) \leq\left\langle f, u_{n}\right\rangle .
$$

Hence, the use of Gronwall's lemma implies $\left\|u_{n}(t)\right\|^{2} \leq A_{T}$ for all $t \leq T$. Moreover, observing (2.28), (6.4) and (6.12), we readily obtain $T_{n}(E)>0$ and

$$
\left\|v_{n}(t)\right\|_{3}^{4} \leq c_{0}\left\|u_{n}(t)\right\|^{2}\left\|\nabla v_{n}(t)\right\|^{2} \leq c_{0} A_{T} \nu_{0} E, \quad t \leq T_{n}(E)
$$

for all $n \geq n_{0}$. So that $2 \mu_{n, 0}-\gamma_{0}\left\|v_{n}\right\|_{3} \geq \mu_{n, 0}$. Integrating (6.17) over the interval $(0, t)$, applying Gronwall's lemma and letting $h \rightarrow 0$, we obtain

$$
\begin{gathered}
\left\|u_{n}^{\prime}(t)\right\|^{2}+\frac{\mu_{n, 0}}{4} \int_{0}^{t}\left\|v_{n}^{\prime}\right\|^{2} d t \\
\leq\left\{\|f(0)-\chi\|^{2}+\int_{0}^{T}\left\{\left\|f^{\prime}\right\|+2\left\|\nu \mu^{\prime}\right\|_{b}^{2}\left\|\sqrt{\mu} \nabla v_{n}\right\|_{a}^{2}+\left\|\sqrt{\nu} g^{\prime}\right\|^{2}\right) d t\right\} \\
\times \exp \left(\int_{0}^{T}\left\|f^{\prime}\right\| d t\right)
\end{gathered}
$$

for all $t \leq T_{n}(E)$ and all $n \geq n_{0}$.

Exactly as in Lemma 6.1 we can derive

$$
\begin{aligned}
\left\|\nabla v_{n}(t)\right\|_{a}^{2} & \leq C_{1} \nu_{0}^{2}\left(\|\nu \nabla \mu(t)\|_{\alpha}^{2}+1\right)\left(\left\|u_{n}^{\prime}(t)\right\|^{2}+\|f(t)\|^{2}+g_{1}^{2}\right. \\
& \left.+\mu_{0}^{2}\left\|\nabla v_{n}(t)\right\|^{2}+\left\|v_{n}(t)\right\|_{\alpha}^{2}\left\|\nabla v_{n}(t)\right\|^{2}\right) .
\end{aligned}
$$


Employing again Gronwall's lemma after substitution of (6.20) into (6.19), we get (6.14), since $\|v\|_{\alpha} \leq\|v\|^{\lambda}\|v\|_{6}^{1-\lambda}$.

Secondly, we suppose (ii) of (6.12) to hold. The use of (2.29) in the LHS of (6.16) implies

$$
\begin{gathered}
\frac{d}{d t}\left\|\delta_{h} u_{n}\right\|^{2}+\frac{1}{4}\left(2 \mu_{n, 0}-\eta\left\|\nabla v_{n}\right\|\right)\left\|\nabla \delta_{h} v_{n}\right\|^{2} \\
\leq\left\|\delta_{h} f_{n}\right\|+\left(1+\frac{2}{n}\right)\left(2\left\|\nu_{n} \delta_{h} \mu\right\|_{b}^{2}\left\|\sqrt{\mu_{n}} \nabla v_{n}\right\|_{a}^{2}+\left\|\sqrt{\nu_{n}} \delta_{h} g\right\|^{2}\right) \\
+\left(\left\|\delta_{h} f\right\|+2 \gamma_{1} \eta^{-3}\left\|\nabla v_{n}\right\|\right)\left\|\delta_{h} u_{n}\right\|^{2},
\end{gathered}
$$

where $\eta^{4}=T$ and we used the inequality:

(6.21) $\left\|\delta_{h} v_{n}\right\|^{2} \leq 2\left\|\delta_{h} u_{n}\right\|^{2}+\frac{2}{n}\left(2\left\|\nu_{n} \delta_{h} \mu\right\|_{b}^{2}\left\|\sqrt{\mu_{n}} \nabla v_{n}\right\|_{a}^{2}+\left\|\sqrt{\nu_{n}} \delta_{h} g\right\|^{2}\right)$,

which is easily derived from (3.14) by observing that

the RHS of $(3.14) \leq \int_{\Omega}\left\{2 \mu\left(t_{i}\right)\left|D\left(v_{j}\right)\right|+g\left(t_{i}\right)\right\}\left(\left|D\left(v_{j}\right)\right|-\left|D\left(v_{i}\right)\right|\right) d x$.

Therefore, we have

$$
\begin{gathered}
\left\|u_{n}^{\prime}(t)\right\|^{2}+\frac{\mu_{n, 0}}{4} \int_{0}^{t}\left\|v_{n}^{\prime}\right\|^{2} d t \\
\leq\left\{\|f(0)-\chi\|^{2}+\int_{0}^{T}\left(\left\|f^{\prime}\right\|+2\left(1+\frac{2}{n}\right)\left\|\nu \mu^{\prime}\right\|_{b}^{2}\left\|\sqrt{\mu} \nabla v_{n}\right\|_{a}^{2}+\left\|\sqrt{\nu} g^{\prime}\right\|^{2}\right) d t\right\} \\
\times \exp \left(\int_{0}^{T}\left\|f^{\prime}\right\| d t+\gamma_{1} \mu_{0}\right)
\end{gathered}
$$

for all $t \leq T_{n}(E)$ and all $n \geq n_{0}$. By the same argument as above we arrive at (6.14).

Q. E. D.

Our next task is to find $E$ such that $T_{n}(E)=T$. From (6.18) it easily follows that

$$
\varphi_{n}\left(t, v_{n}(t)\right)^{2} \leq 2\left\|u_{n}(t)\right\|^{2}\left(\|f(t)\|^{2}+\left\|u_{n}^{\prime}(t)\right\|^{2}\right) .
$$

Accordingly, if $E$ is chosen so that

$$
9 A_{T}\left(\max _{0 \leq t \leq T}\|f(t)\|^{2}+I_{T}\right)+9 A_{T} J_{T}\left(\mu_{0} E+A_{T}^{\lambda} \mu_{0}^{\lambda-2} E^{2-\lambda}\right)<E^{2},
$$

then we can derive from (6.22) and Proposition 6.1 that

$$
\mu_{0}\left\|\nabla v_{n}(t)\right\|^{2} \leq \sqrt{9 / 2} \varphi_{n}\left(t, v_{n}(t)\right)<E
$$


for all $t \leq T_{n}(E)$ and all $n \geq n_{0}$. Hence, it is concluded that $T_{n}(E)=T$. In fact, this contradicts the definition (6.13) if $T_{n}(E)<T$. For the sake of simplicity we write

$$
\text { (6.23) as } B_{0}+B_{1} E+B_{2} E^{2-\lambda}<E^{2} \text {. }
$$

Set

$$
E_{1}=\left(2 B_{2}\right)^{1 / \lambda} \text { and } E_{2}=2 B_{1}+\sqrt{2 B_{0}} .
$$

Then, $B_{2} E_{1}^{2-\lambda}=E_{1}^{2} / 2$ and $B_{0}+B_{1} E_{2} \leq E_{2}^{2} / 2$. It is easily verified that $E_{T}=E_{1}+E_{2}$ satisfies (6.23).

The inequality $\mu_{0}\left\|\nabla u_{0}\right\|^{2}<E_{T}$ is then trivial. Making use of the compactness argument, we thus arrive at (2.32). Evidently, $u$ is a solution of (2.25)-(2.26). Moreover, with the aid of Lemma 6.2 we can prove that (2.33) are bounded. Let $l$ be the integer mentioned in Lemma 6.2. Then, (6.11) implies

$$
\|\nabla u\|_{q} \leq c_{l}\left\{P^{l}(\|\nabla u\|+\|\nu \pi\|)+\frac{P^{l}-1}{P-1} Q_{r}\right\},
$$

where $P(t)$ is bounded and $Q_{r}(t)$ is the sum of the bounded function and $\left\|f(t)-u^{\prime}(t)\right\|_{r}$. If $2 \leq q \leq 6$, then $6 / 5 \leq r \leq 2$. We now suppose $q>6$. Then, $2<r<3$. By (1.10) and Sobolev's inequality we have

$$
\left\|u^{\prime}\right\|_{r} \leq \text { const. }\left\|u^{\prime}\right\|^{1-\delta}\left\|\nabla u^{\prime}\right\|^{\delta}
$$

where $\delta=3(1 / 2-1 / r)$ and $1 / r=1 / q+1 / 3$. Therefore, $\|\nabla u\|_{q}^{p}$ is integrable for $p=2 / \delta$, which completes the proof of the fact mentioned above. The uniqueness easily follows from (ii) of Corollary 2.

\section{REFERENCES}

[1] G. Astarita and G. Marrucci, Principles of Non-Newtonian Fluid Mechanics, McGraw-Hill, London, 1974.

[2] M. S. Berger, Nonlinear and Functional Analysis, Academic Press, New York-San Francisco-London, 1977.

[ 3 ] H. Brezis, Problèmes unilateraux, J. Math. Pures Appl., 51 (1972), 1-168.

[4] L. Cattabriga, Su un problema al contorno relativo al sistema di equazioni di Stokes, Rend. Mat. Univ. Padova, 31 (1961), 308-340.

[5] G. Duvaut and J. L. Lions, Ecoulement d'un fluide rigide viscoplastique incompressible, C. R. Acad. Sc. Paris, 270 (1970), 58-61.

[6] — Les inéquations en méchanique et en physique (English translation), Springer-Verlag, Berlin-Heiderberg-New York, 1976.

[ 7 ] J. U. Kim, On the Cauchy problem associated with the motion of a Bingham fluid in 
the plane, Trans. Amer. Math. Soc., 298 (1986), 371-400.

[8] - On the initial boundary value problem for a Bingham fluid in a three dimensional domain, Trans. Amer. Math. Soc., 304 (1987), 751-770.

[9] J. L. Lions, Quelque méthodes de résolution des problèmes aux limites non linéaires, Dunod/Gauthier-Villars, Paris, 1969

[10] K. Masuda, Weak solutions of Navier-Stokes equations, Tôhoku Math. J., 36 (1984), 623-646.

[11] T. Miyakawa and H. Sohr, On energy inequality, smoothness and large time behavior in $L^{2}$ for weak solutions of the Navier-Stokes equations in exterior domains, Math. Z., 199 (1988), 455-478.

[12] P. P. Mosolov and V. P. Mjasnikov, On the correctness of boundary value problems in the mechanics of continuous media, Math. USSR Sbornik, 17 (1972), 257-268.

[13] J. Naumann and Wulst, On evolution inequalities of Bingham type in three dimensions, I, J. Math. Anal. Appl., 68 (1979), 211-227.

[14] - - On evolution inequalities of Bingham type in three dimensions, II, J. Math. Anal. Appl., 70 (1979), 309-325.

[15] M. Renardy, Dense imbedding of test funtions in certain function spaces, Trans. Amer. Math. Soc., 298 (1986), 241-243.

[16] M. J. Strauss, Variations of Korn's and Sobolev's inequalities, Berkeley symposium on partial differential equations, A.M.S. Symposia, 23 (1971).

[17] R. Temam, Navier-Stokes equations, North-Holland, 1977.

[18] K. Yosida, Functional Analysis, Springer-Verlag, Berlin-Heiderberg-New York, 1966

\author{
Department of Applied Physics \\ School of Engineering \\ Nagoya University, Nagoya 464-01 \\ JAPAN
}

